\title{
Accelerated Gradient Descent Methods for the Uniaxially Constrained Landau-de Gennes Model
}

\author{
Edison E. Chukwuemeka ${ }^{1}$ and Shawn W. Walker ${ }^{2, *}$ \\ ${ }^{1}$ Department of Mechanical Engineering, Louisiana State University, Baton Rouge, \\ LA 70803, USA \\ ${ }^{2}$ Department of Mathematics and Center for Computation and Technology (CCT), Louisiana \\ State University, Baton Rouge, LA 70803, USA
}

Received 12 March 2021; Accepted (in revised version) 25 August 2021

\begin{abstract}
This paper illustrates the efficacy of using accelerated gradient descent schemes for minimizing a uniaxially constrained Landau-de Gennes model for nematic liquid crystals. Three (alternating direction) minimization schemes are applied to a structure preserving finite element discretization of the uniaxial model: a standard gradient descent method, the "heavy-ball" method, and Nesterov's method. The performance of the schemes is measured in terms of the number of iterations required to obtain the equilibrium state, as well as the total computational time (wall time).

The numerical experiments clearly show that the accelerated gradient descent schemes reduce the number of iterations and computational time significantly, despite the hard uniaxial constraint that is not "smooth" when defects are present. Moreover, our results show that accelerated schemes are not hindered when combined with an alternating direction minimization algorithm and are easy to implement.
\end{abstract}

AMS subject classifications: 65N30, 49M25, 35J70

Key words: Liquid crystals, Landau-de Gennes, uniaxial, heavy-ball method, Nesterov's method.

\section{Introduction}

Liquid crystals (LCs) are important in many emerging technologies $[17,28]$. They are easily actuated by optical effects $[8,15,20,23,57]$, electric/magnetic $[2,10,51]$, and mechanical forces [7, 18,50,69], which has yielded various devices, e.g., electronic shutters [22], novel types of lasers [14,24], dynamic shape control of elastic bodies [11,62], and others $[31,37,58,61,63]$.

LC models use an order parameter that represents the statistical average of the orientation of the LC molecules [59]. The Landau-de Gennes (LdG) model is popular and

*Corresponding author.

Emails: echukw3@lsu.edu (E. E. Chukwuemeka), walker@math.lsu.edu (S. W. Walker) 
uses a tensor-valued function $\mathbf{Q}$ to model the orientational state of the LC material. In particular, the eigenframe of $\mathbf{Q}$ yields information about the distribution of LC molecules. Moreover, the energy functional (of $\mathbf{Q}$ ) for the LC material involves an elastic contribution and a bulk potential. Equilibrium LC states are minimizers of the energy functional.

This paper is concerned with a specific variant of the LdG model where uniaxiality is enforced as a hard constraint (see Section 3). When defects are present, i.e., when the order parameter $\mathbf{Q}$ vanishes, this constraint is not smooth, which makes using standard second order methods difficult (e.g., a monolithic Newton method). Of course, standard gradient descent methods can be used and are robust, but can be extremely slow to converge to a minimizer. Therefore, we investigate alternative methods for minimizing the energy functional, so-called accelerated gradient descent methods, e.g., the "heavy-ball" method [47] and Nesterov's method [40]. Our numerical experiments clearly show that both the heavy-ball method and Nesterov method are significantly better than standard gradient descent, despite the presence of the non-smooth uniaxial constraint.

Although many numerical methods and implementations exist for the standard LdG model, e.g., $[5,6,9,16,30,49,66,67]$, to the best of our knowledge, accelerated gradient methods for LC simulations, in general, are not typically used. There are, of course, examples of accelerated methods used for other types of PDEs. For example, in [25], they consider a phase field crystal model. In that work, they establish various analytical results, including convergence guarantees for their accelerated method. However, the model we consider has an elliptic degeneracy (see Remark 3.2, as well as $[9,44]$ ), which is a major analytical difficulty that does not appear in the model considered by [25]. Indeed, it is not clear how to derive a convergence guarantee that accounts for the elliptic degeneracy and the associated non-smooth constraint. Our main contribution is to demonstrate that well-known acceleration techniques are still effective in speeding up convergence to a minimizer for energy functionals that are degenerate with non-smooth constraints.

This paper is organized as follows. A review of the Landau-de Gennes model using the one constant version is presented in Section 2. The Landau-de Gennes model is reduced to the uniaxially constrained model in Section 3. A high level description of the minimization schemes is reviewed in Section 4, and then applied to minimizing the Landau-de Gennes energy in Section 5. Numerical experiments are conducted in Section 6 to illustrate the performance of the minimization schemes. We close with some remarks in Section 7.

\section{The Landau-de Gennes model}

We briefly review the Landau-de Gennes model $[17,55]$. Let $\Omega \subset \mathbb{R}^{d}$, with $d=2,3$, be the domain of the LC material (assume $\Omega$ is Lipschitz). The order parameter $\mathbf{Q}$ is a tensorvalued function $Q: \Omega \rightarrow \Lambda$, where

$$
\mathbf{\Lambda}:=\left\{\mathbf{A} \in \mathbb{R}^{d \times d} \mid \mathbf{A}=\mathbf{A}^{T}, \operatorname{tr}(\mathbf{A})=0\right\} .
$$


The "one-constant" energy functional for $\mathbf{Q}$ is

$$
E_{\mathrm{LdG}}[\mathbf{Q}]:=\frac{1}{2} \int_{\Omega}|\nabla \mathbf{Q}|^{2} d \mathbf{x}+\frac{1}{\epsilon_{\mathrm{dw}}^{2}} \int_{\Omega} \psi_{\mathrm{LdG}}(\mathbf{Q}) d \mathbf{x},
$$

where $\epsilon_{\mathrm{dw}}>0$ is a material parameter (nematic correlation length-scale), and $\psi_{\mathrm{LdG}}$ is a "bulk" (thermotropic) potential.

Note:

$$
|\nabla \mathbf{Q}|^{2}=\sum_{i, j, k}\left(\partial_{k} Q_{i j}\right)^{2}
$$

More complicated models can also be considered [17,36,55].

The bulk potential $\psi_{\mathrm{LdG}}$ is a double-well type of function that captures the nematic to isotropic phase transition of nematic LCs. Mathematically, it tries to confine the eigenvalues of $\mathbf{Q}$ to the physically meaningful range $\lambda_{i} \in[-1 / d, 1-1 / d]$, where the simplest form is given by

$$
\psi_{\mathrm{LdG}}(\mathbf{Q})=K+\frac{A}{2} \operatorname{tr}\left(\mathbf{Q}^{2}\right)-\frac{B}{3} \operatorname{tr}\left(\mathbf{Q}^{3}\right)+\frac{C}{4}\left(\operatorname{tr}\left(\mathbf{Q}^{2}\right)\right)^{2} \geq 0,
$$

where $A, B, C$ are material parameters such that $A$ may be positive or negative, and $B$, $C$ are positive; $K$ is a convenient constant. Critical points of $\psi_{\mathrm{LdG}}$ are either isotropic or uniaxial Q-tensors [33]; indeed, $A \leq 0$ and $B, C>0$ favors uniaxial states over isotropic states. Note: in two dimensions, $\operatorname{tr}\left(\mathbf{Q}^{3}\right)=0$, because $\mathbf{Q}^{2}=\frac{s^{2}}{4} \mathbf{I}$. Hence, $B$ is irrelevant when $d=2$.

One seeks to minimize (2.2) over $\mathbf{Q}$ in $H^{1}(\Omega ; \boldsymbol{\Lambda})$ with suitable boundary conditions, e.g., Dirichlet. When $d=3$, a simple argument [55] shows that minimizers of $\mathbf{Q} \mapsto$ $\int_{\Omega} \psi_{\mathrm{LdG}}(\mathbf{Q})$ have a uniaxial form, i.e.,

$$
\mathbf{Q}=s\left(\mathbf{n} \otimes \mathbf{n}-\frac{1}{3} \mathbf{I}\right),
$$

where $\mathbf{n} \in \mathbb{S}^{2}$ and $s \in[-1 / 2,1]$. However, general minimizers of (2.2) are not necessarily uniaxial; they are typically biaxial $[29,46,54]$ :

$$
\mathbf{Q}=-s_{1}\left(\mathbf{n}_{1} \otimes \mathbf{n}_{1}-\frac{1}{3} \mathbf{I}\right)-s_{2}\left(\mathbf{n}_{2} \otimes \mathbf{n}_{2}-\frac{1}{3} \mathbf{I}\right)
$$

where $\mathbf{n}_{1}, \mathbf{n}_{2} \in \mathbb{S}^{2}, \mathbf{n}_{1} \cdot \mathbf{n}_{2}=0$, and $s_{1}=-2 \lambda_{1}-\lambda_{2}, s_{2}=-\lambda_{1}-2 \lambda_{2}$. Note: when $d=2, \mathbf{Q}$ always has the form

$$
\mathbf{Q}=s\left(\mathbf{n} \otimes \mathbf{n}-\frac{1}{2} \mathbf{I}\right) \text {. }
$$

But this brings up a modeling issue. Some types of LCs do exhibit biaxiality [19], such as lyotropic LCs (see [65]). But thermotropic LCs do not typically exhibit a biaxial phase in experiments; Indeed, a biaxial phase was only first reported in $[1,32,48]$. Therefore, it is plausible that uniaxiality should be an inherent part of the model for some types 
of LC systems. In this paper, we consider the LdG model with uniaxiality enforced as a hard constraint (see Section 3). The main contribution here is to describe alternative minimization schemes to compute energy minimizers with such a non-smooth constraint (see Section 4).

\section{The uniaxially constrained Q-model}

This section describes the mathematical formulation of the minimization problem for the one-constant Landau-deGennes energy $E_{\text {LdG }}$ under the uniaxiality constraint (2.4) (Section 3.1). The model we obtain has similarities with the Ericksen model [9,43-45], but it has the advantage of allowing for non-orientable minimizers that exhibit half-integer order defects. This model is mainly of interest when $d=3$, since when $d=2, \mathbf{Q}$ necessarily has the form of a uniaxial tensor. A small advantage of this approach is that it yields a model with fewer variables; the standard LdG model involves a non-linear system with five coupled variables in 3-D [30,49,66,68]. In addition, the uniaxial model provides a way to easily control the eigenvalues of $\mathbf{Q}$, which the standard model does not. For more information on the uniaxial model and discretization (Section 3.2), see [9].

Remark 3.1. Uniaxial models arise in a small elastic constant limit. In [34], the oneconstant model is studied (2.2) with a small bulk coefficient $\epsilon_{\mathrm{dw}}$ (which is equivalent to a small elastic constant). Under suitable boundary conditions, in the limit $\epsilon_{\mathrm{dw}} \rightarrow 0$, Landaude Gennes minimizers converge to minimizers of the Oseen-Frank energy. The analysis in [34] is refined in [41], where the dependence of the difference between the solution to both models with respect to $\epsilon_{\mathrm{dw}}$ is analyzed.

\subsection{Theoretical background}

For a uniaxially constrained Q-tensor as in (2.4), set $\boldsymbol{\Theta}=\mathbf{n} \otimes \mathbf{n}$ and minimize (2.2) with respect to $s$ and $\boldsymbol{\Theta}$. Define the space

$$
L^{d-1}=\left\{\mathbf{A} \in \mathbb{R}^{d \times d}: \text { there exists } \mathbf{n} \in \mathbb{S}^{d-1}, \mathbf{A}=\mathbf{n} \otimes \mathbf{n}\right\},
$$

which is isomorphic to the real projective space $\mathbf{R P}^{d-1}$ by the $\operatorname{map} \mathbf{n} \otimes \mathbf{n} \longmapsto\{\mathbf{n},-\mathbf{n}\}$. So the uniaxially constrained LdG model accounts for the molecular direction (i.e., a line segment) but not the orientation (i.e., a vector).

Since

$$
\nabla \mathbf{Q}=\nabla s \otimes\left(\boldsymbol{\Theta}-\frac{1}{d} \mathbf{I}\right)+s \nabla \mathbf{\Theta}
$$

we get

$$
|\nabla \mathbf{Q}|^{2}=|\nabla s|^{2}\left|\boldsymbol{\Theta}-\frac{1}{d} \mathbf{I}\right|^{2}+s^{2}|\nabla \boldsymbol{\Theta}|^{2}+2 s\left[\nabla s \otimes\left(\boldsymbol{\Theta}-\frac{1}{d} \mathbf{I}\right)\right]: \nabla \boldsymbol{\Theta} .
$$


A simple calculation gives

$$
\left|\boldsymbol{\Theta}-\frac{1}{d} \mathbf{I}\right|^{2}=\frac{d-1}{d} \quad \text { and } \quad\left[\nabla s \otimes\left(\boldsymbol{\Theta}-\frac{1}{d} \mathbf{I}\right)\right]: \nabla \boldsymbol{\Theta}=0,
$$

so that

$$
|\nabla \mathbf{Q}|^{2}=\frac{d-1}{d}|\nabla s|^{2}+s^{2}|\nabla \Theta|^{2}
$$

Note the following straightforward equalities:

$$
\begin{array}{llll}
\text { for } d=2: & (1 / 2) s^{2}=\operatorname{tr}\left(\mathbf{Q}^{2}\right), & 0=\operatorname{tr}\left(\mathbf{Q}^{3}\right), & (1 / 4) s^{4}=\left(\operatorname{tr}\left(\mathbf{Q}^{2}\right)\right)^{2}, \\
\text { for } d=3: & (2 / 3) s^{2}=\operatorname{tr}\left(\mathbf{Q}^{2}\right), & (2 / 9) s^{3}=\operatorname{tr}\left(\mathbf{Q}^{3}\right), & (4 / 9) s^{4}=\left(\operatorname{tr}\left(\mathbf{Q}^{2}\right)\right)^{2} .
\end{array}
$$

Therefore, the one-constant energy for the uniaxially constrained Q-tensor model is obtained by plugging (3.2) into (2.2) to get

$$
\begin{aligned}
& E_{\mathrm{LdG}}[\mathbf{Q}]=E_{\text {uni }}[s, \boldsymbol{\Theta}]:=E_{\text {uni }-\mathrm{m}}[s, \boldsymbol{\Theta}]+E_{\mathrm{LdG}, \text { bulk }}[s], \\
& E_{\text {uni }-\mathrm{m}}[s, \boldsymbol{\Theta}]:=\frac{1}{2}\left(\frac{d-1}{d} \int_{\Omega}|\nabla s|^{2} d x+\stackrel{\circ}{E}_{\text {uni }}[s, \boldsymbol{\Theta}]\right), \\
& \stackrel{\circ}{E}_{\text {uni }}[s, \Theta]:=\int_{\Omega} s^{2}|\nabla \Theta|^{2} d x, \quad E_{\mathrm{LdG}, \text { bulk }}[s]:=\frac{1}{\epsilon_{\mathrm{dw}}^{2}} \int_{\Omega} \psi_{\mathrm{LdG}}(s) d x,
\end{aligned}
$$

where, with some abuse of notation, we write

$$
\psi_{\mathrm{LdG}}(s):=\psi_{\mathrm{LdG}}(\mathbf{Q}) .
$$

Remark 3.2. The functional $\stackrel{\circ}{\text { uni }}[s, \boldsymbol{\Theta}]$ has the integrand $s^{2}|\nabla \boldsymbol{\Theta}|^{2}$, which is elliptic degenerate because $s$ may vanish. This is a direct consequence of enforcing the uniaxial constraint (2.4) (see [9]).

The admissible class for minimization becomes more clear upon using the following change of variable $\mathbf{U}=s \boldsymbol{\Theta}$ and rewrite

$$
E_{\text {uni-m }}[s, \mathbf{\Theta}]=\widetilde{E}_{\text {uni }-\mathrm{m}}[s, \mathbf{U}]:=\frac{1}{2}\left(-\frac{1}{d} \int_{\Omega}|\nabla s|^{2} d x+\int_{\Omega}|\nabla \mathbf{U}|^{2} d x\right) .
$$

Since the degree of orientation $s$ needs to satisfy $s \in\left[-\frac{1}{d-1}, 1\right]$, we define the admissible class as

$$
\mathcal{A}_{\text {uni }}:=\left\{(s, \boldsymbol{\Theta}) \in H^{1}(\Omega) \times\left[L^{\infty}(\Omega)\right]^{d \times d}:(s, \mathbf{U}, \boldsymbol{\Theta}) \text { satisfies (3.7), with } \mathbf{U} \in\left[H^{1}(\Omega)\right]^{d}\right\},
$$

with the structural condition

$$
-\frac{1}{d-1} \leq s \leq 1, \quad \mathbf{U}=s \boldsymbol{\Theta}, \quad \boldsymbol{\Theta} \in L^{d-1} \text { a.e. in } \Omega .
$$


In order to enforce boundary conditions on $(s, \boldsymbol{\Theta})$, on different parts of the boundary, we introduce auxiliary functions $g: \Omega \rightarrow\left(-\frac{1}{d-1}, 1\right)$ and $\mathbf{M}: \Omega \rightarrow L^{d-1}$ and define the restricted admissible class

$$
\mathcal{A}_{\text {uni }}(g, \mathbf{M}):=\left\{(s, \boldsymbol{\Theta}) \in \mathcal{A}_{\text {uni }}:\left.s\right|_{\Gamma_{s}}=g,\left.\boldsymbol{\Theta}\right|_{\Gamma_{\boldsymbol{\Theta}}}=\mathbf{M}\right\},
$$

where $\Gamma_{s}$ and $\Gamma_{\boldsymbol{\Theta}}$ are open subsets of $\partial \Omega$ on which to enforce Dirichlet conditions for $s$ and $\Theta$ (respectively).

See [9] for more details on the function spaces and theoretical details. In this paper, we are only concerned with the minimization scheme for the discrete problem (Section 4).

\subsection{Discretization}

We discretize $\Omega \subset \mathbb{R}^{d}$ with a conforming simplicial shape-regular triangulation $\mathcal{T}_{h}=\left\{T_{i}\right\}$ (ignoring any geometric error caused by domain approximation), with nodes (vertices) $\mathcal{N}_{h}$. Moreover, we make the following critical assumption with regard to our finite element scheme.

Assumption 3.1. We assume throughout that the simplicial mesh $\mathcal{T}_{h}$ is weakly-acute in the sense of $[4,13,26,27,56]$. This is necessary because of the form of our discrete energy (3.13).

Next, define continuous linear Lagrange finite element spaces on $\Omega$, i.e., let $s_{h}$ in $S_{h}$, and $\boldsymbol{\Theta}_{h}$ in $\mathbb{T}_{h}$, be the finite element approximations of $s$ and $\boldsymbol{\Theta}$, where

$$
\begin{aligned}
& \mathbb{S}_{h}:=\left\{z_{h} \in H^{1}(\Omega):\left.z_{h}\right|_{T} \in \mathcal{P}_{1}(T), \forall T \in \mathcal{T}_{h}\right\}, \\
& \overline{\mathbb{U}}_{h}:=\left\{\mathbf{U}_{h} \in\left[H^{1}(\Omega)\right]^{d \times d}:\left.\mathbf{U}_{h}\right|_{T} \in \mathcal{P}_{1}(T), \forall T \in \mathcal{T}_{h}\right\}, \\
& \mathbb{T}_{h}:=\left\{\boldsymbol{\Theta}_{h} \in \overline{\mathbb{U}}_{h}: \boldsymbol{\Theta}_{h}\left(x_{i}\right) \in L^{d-1}, \forall x_{i} \in \mathcal{N}_{h}\right\},
\end{aligned}
$$

where $\mathbb{T}_{h}$ imposes the rank-one, unit norm constraint only at the vertices of the mesh. Dirichlet boundary conditions are included via the following discrete spaces:

$$
\mathbb{S}_{h}\left(\Gamma_{s}, g_{h}\right):=\left\{s_{h} \in \mathbb{S}_{h}:\left.s_{h}\right|_{\Gamma_{s}}=g_{h}\right\}, \quad \mathbb{T}_{h}\left(\Gamma_{\boldsymbol{\Theta}}, \mathbf{M}_{h}\right):=\left\{\boldsymbol{\Theta}_{h} \in \mathbb{T}_{h}:\left.\Theta_{h}\right|_{\Gamma_{\boldsymbol{\Theta}}}=\mathbf{M}_{h}\right\},
$$

where $g_{h}:=I_{h} g$ and $\mathbf{M}_{h}:=I_{h} \mathbf{M}$ are the discrete Dirichlet data. This yields the discrete admissible class with boundary conditions:

$$
\mathcal{A}_{\text {uni }}^{h}\left(g_{h}, \mathbf{M}_{h}\right):=\left\{\left(s_{h}, \boldsymbol{\Theta}_{h}\right) \in \mathbb{S}_{h}\left(\Gamma_{s}, g_{h}\right) \times \mathbb{T}_{h}\left(\Gamma_{\boldsymbol{\Theta}}, \mathbf{M}_{h}\right):\left(s_{h}, \mathbf{U}_{h}, \boldsymbol{\Theta}_{h}\right) \text { satisfies (3.12) }\right\},
$$

where

$$
\mathbf{U}_{h}=I_{h}\left(s_{h} \boldsymbol{\Theta}_{h}\right) \in \overline{\mathbb{U}}_{h}, \quad-\frac{1}{d-1} \leq s_{h} \leq 1 \text { in } \Omega, \quad \text { and } \quad \boldsymbol{\Theta}_{h}\left(x_{i}\right) \in L^{d-1}, \quad \forall x_{i} \in \mathcal{N}_{h},
$$


is called the discrete structural condition of $\mathcal{A}_{\mathrm{uni}}^{h}$.

The discrete version of $E_{\mathrm{uni}-\mathrm{m}}[s, \boldsymbol{\Theta}]$ is derived in [9]. We set $\delta_{i j} s_{h}:=s_{h}\left(x_{i}\right)-s_{h}\left(x_{j}\right)$, and $\delta_{i j} \boldsymbol{\Theta}_{h}:=\boldsymbol{\Theta}_{h}\left(x_{i}\right)-\boldsymbol{\Theta}_{h}\left(x_{j}\right)$ for $x_{i}, x_{j} \in \mathcal{N}_{h}$, and define the main part of the discrete energy to be

$$
E_{\mathrm{uni}-\mathrm{m}}^{h}\left[s_{h}, \boldsymbol{\Theta}_{h}\right]:=\frac{d-1}{4 d} \sum_{i, j=1}^{n} k_{i j}\left(\delta_{i j} s_{h}\right)^{2}+\frac{1}{4} \sum_{i, j=1}^{n} k_{i j}\left(\frac{s_{h}\left(x_{i}\right)^{2}+s_{h}\left(x_{j}\right)^{2}}{2}\right)\left|\delta_{i j} \boldsymbol{\Theta}_{h}\right|^{2},
$$

where, because of Assumption 3.1, there holds

$$
k_{i j}:=-\int_{\Omega} \nabla \phi_{i} \cdot \nabla \phi_{j} d x \geq 0 \text { for all } i \neq j,
$$

where $\phi_{i}$ is the standard "hat" function associated with node $x_{i} \in \mathcal{N}_{h}$. The first term in (3.13) corresponds to

$$
\frac{1}{2} \sum_{i, j=1}^{n} k_{i j}\left(\delta_{i j} s_{h}\right)^{2}=\int_{\Omega}\left|\nabla s_{h}\right|^{2} d x
$$

while the second term is a first order approximation of $\frac{1}{2} \int_{\Omega} s^{2}|\nabla \Theta|^{2} d x$. For convenience, we shall denote

$$
\stackrel{\circ}{\mathrm{uni}}_{\mathrm{uni}}^{h}\left[s_{h}, \mathbf{\Theta}_{h}\right]:=\frac{1}{2} \sum_{i, j=1}^{n} k_{i j}\left(\frac{s_{h}\left(x_{i}\right)^{2}+s_{h}\left(x_{j}\right)^{2}}{2}\right)\left|\delta_{i j} \mathbf{\Theta}_{h}\right|^{2} .
$$

The bulk energy is discretized in the obvious way:

$$
E_{\mathrm{LdG}, \text { bulk }}^{h}\left[s_{h}\right]:=\frac{1}{\epsilon_{\mathrm{dw}}^{2}} \int_{\Omega} \psi_{\mathrm{LdG}}\left(s_{h}\right) d x .
$$

With the notation introduced above, the formulation of the discrete problem reads as follows. Find $\left(s_{h}, \boldsymbol{\Theta}_{h}\right) \in \mathbb{S}_{h}\left(\Gamma_{s}, g_{h}\right) \times \mathbb{T}_{h}\left(\Gamma_{\boldsymbol{\Theta}}, \mathbf{M}_{h}\right)$ such that the following energy is minimized:

$$
E_{\mathrm{uni}}^{h}\left[s_{h}, \boldsymbol{\Theta}_{h}\right]:=E_{\mathrm{uni}-\mathrm{m}}^{h}\left[s_{h}, \boldsymbol{\Theta}_{h}\right]+E_{\mathrm{LdG}, \mathrm{bulk}}^{h}\left[s_{h}\right] .
$$

The $\Gamma$-convergence of this discrete energy was shown in [9].

\section{Review of gradient based minimization schemes}

We give a general discussion of gradient descent and accelerated gradient descent methods. We apply these schemes to the uniaxial Q-model in Section 5. Our numerical experiments in Section 6 will show that both the heavy-ball method and Nesterov method perform significantly faster than the more standard approach in [9], especially when coupled with an exact line-search. 


\subsection{Classical gradient descent}

Let $X$ be a Banach space and $f: X \rightarrow \mathbb{R}$ a differentiable function that is bounded below, so has a minimizer. Given the current guess $x_{k}$, the simplest form of gradient descent is to first compute a descent direction $p_{k}: a\left(p_{k}, v\right)=-\delta f\left[x_{k}\right](v)$, for all $v \in X$, where $a(\cdot, \cdot): X \times X \rightarrow \mathbb{R}$ is some appropriate, coercive bilinear form that is an inner product on $X$. Then, update the guess: $x_{k+1}:=x_{k}+\alpha p_{k}$, for some step size $\alpha>0$. This procedure is iterated until some convergence criteria is achieved, such as

$$
\left\|\delta f\left[x_{k}\right]\right\|_{X^{*}}<\varepsilon_{\text {tol }}
$$

for some tolerance $\varepsilon_{\text {tol }}>0$.

Choosing $\alpha$ appropriately, or using a line-search [42], yields a robust method but is usually quite slow to converge. The form of $f$ can force the step size to be very small in order to ensure decrease of $f$, which is the case for the energy functional in (3.17). The standard approach can be improved by choosing $a(\cdot, \cdot)$ so that it is "matched" to $\delta f$, or by making the descent solve "more implicit." The later approach is motivated by minimizing movements [35], and is described more in the next section.

\subsection{Accelerated gradient descent}

Algorithm 4.1 describes the minimizing movements strategy for gradient descent. It also includes a "momentum" term, known as the "heavy-ball" method (see [47] for the original method).

Setting $\beta=0$, since $p_{k}$ minimizes $G_{k}[\cdot]$, choosing $\alpha=1$, we see that

$$
f\left[x_{k+1}\right]=f\left[x_{k}+p_{k}\right]<(1 / 2)\left\|p_{k}\right\|_{a}^{2}+f\left[x_{k}+p_{k}\right]=G_{k}\left[p_{k}\right] \leq G_{k}[0]=f\left[x_{k}\right],
$$

i.e., each step is guaranteed to decrease the functional. Choosing $\gamma>0$ sufficiently small ensures that $G_{k}[\cdot]$ is convex.

If $\beta>0$, then this adds a bias term that influences the current search direction to follow the old search direction (i.e., an inertial term). It has been demonstrated, both theoretically and in practice, that the heavy-ball method can alleviate the "zig-zagging" behavior of classical gradient descent [47]. Of course, if $\beta>0$, then strict decrease of the function is not guaranteed, unless a line-search is used.

Another accelerated gradient descent method is the well-known Nesterov method [40] (see Algorithm 4.2). One can think of this method as a more optimized version of the heavy-ball method.

\section{Accelerated descent schemes for the uniaxial Q-model}

The energy functional $E_{\text {uni }}^{h}\left[s_{h}, \boldsymbol{\Theta}_{h}\right]$ in (3.17) is non-convex. However, fixing either of the variables, and linearizing with respect to the other variable, yields a simple quadratic 
Algorithm 4.1 Minimizing movement gradient descent with momentum term.

Set a tolerance $\varepsilon_{\text {tol }}>0$, initial guess $x_{0} \in X$, and set $k:=0$; choose $\gamma>0$ and $\beta \geq 0$. For $k \geq 0$, do the following:

1. Define the auxiliary function

$$
G_{k}[p]:=(1 / 2)\|p\|_{a}^{2}+\gamma f\left[x_{k}+p\right]-\beta a\left(p_{k-1}, p\right) .
$$

2. Compute search direction. Let $p_{k} \in X$ be the minimizer of $G_{k}[\cdot]$, i.e., $p_{k} \in X$ solves the following variational problem

$$
a\left(p_{k}, v\right)=-\gamma \delta f\left[x_{k}+p_{k}\right](v)+\beta a\left(p_{k-1}, v\right) \quad \text { for all } v \in X,
$$

where $a(\cdot, \cdot): X \times X \rightarrow \mathbb{R}$ is a chosen bilinear form (i.e., inner product) on $X$. This yields a descent direction $p_{k}$.

3. Update: $x_{k+1}:=x_{k}+\alpha p_{k}$, for some step size $\alpha>0$. Replace $k \leftarrow k+1$.

4. If $\left|f\left[x_{k+1}\right]-f\left[x_{k}\right]\right|<\varepsilon_{\text {tol }}$, then stop; else, return to Step 1 .

\section{Algorithm 4.2 Nesterov's gradient descent.}

Set a tolerance $\varepsilon_{\text {tol }}>0$, initial guess $x_{0} \in X, x_{-1}:=x_{0}, a_{-1}:=1$, and set $k:=0$; choose $\gamma>0$. For $k \geq 0$, do the following:

1. Compute auxiliary point. Let $a_{k}=\left(1+\sqrt{4 a_{k-1}^{2}+1}\right) / 2$, and set $\zeta_{k}=\left(a_{k-1}-1\right) / a_{k}$. Then, compute $y_{k}=x_{k}+\zeta_{k}\left(x_{k}-x_{k-1}\right)$.

2. Compute search direction. Let $p_{k} \in X$ solve the following variational problem

$$
a\left(p_{k}, v\right)=-\gamma \delta f\left[y_{k}+p_{k}\right](v) \quad \text { for all } v \in X,
$$

where $a(\cdot, \cdot): X \times X \rightarrow \mathbb{R}$ is a chosen bilinear form (i.e., inner product) on $X$. This yields a descent direction $p_{k}$.

3. Update: $x_{k+1}:=y_{k}+\alpha p_{k}$, for some step size $\alpha>0$. Replace $k \leftarrow k+1$.

4. If $\left|f\left[x_{k+1}\right]-f\left[x_{k}\right]\right|<\varepsilon_{\text {tol }}$, then stop; else, return to Step 1.

minimization to solve. Therefore, we use an alternating direction method to find minimizers of $E_{\mathrm{uni}}^{h}\left[s_{h}, \boldsymbol{\Theta}_{h}\right]$. 


\subsection{Tangential variations}

Any minimization strategy must account for the constraint $\boldsymbol{\Theta}_{h}\left(x_{i}\right) \in L^{d-1}$ for all $x_{i} \in \mathcal{N}_{h}$, in (3.12). We do this by computing variational derivatives that preserve the constraint to first order, i.e., we take variations tangential to the constraint manifold. Since $\boldsymbol{\Theta} \in L^{d-1}$ is characterized by $\mathbf{\Theta}=\mathbf{n} \otimes \mathbf{n}$, we first do a Taylor expansion of $\mathbf{n} /|\mathbf{n}|$ in the direction of $\mathbf{v}$, where $\mathbf{v} \cdot \mathbf{n}=0$ [59], i.e.,

$$
\mathbf{f}(\mathbf{v}):=\frac{\mathbf{n}+\mathbf{v}}{|\mathbf{n}+\mathbf{v}|} \Rightarrow \mathbf{f}(\mathbf{v})=\mathbf{n}+\mathbf{v}-\frac{1}{2}|\mathbf{v}|^{2} \mathbf{n}+o\left(|\mathbf{v}|^{2}\right) .
$$

The first variation of $\mathbf{n} \in \mathrm{S}^{d-1}$, in the direction $\mathbf{v}$, is then

$$
\delta \mathbf{n}(\mathbf{v}):=\left.\frac{d}{d \epsilon} \mathbf{f}(\epsilon \mathbf{v})\right|_{\epsilon=0}=\mathbf{v} \in T_{\mathbf{n}} \mathrm{S}^{d-1}
$$

(the tangent space of $S^{d-1}$ at $\left.\mathbf{n}\right)$. Expanding $(\mathbf{n} /|\mathbf{n}|) \otimes(\mathbf{n} /|\mathbf{n}|)$ we get

$$
\frac{\mathbf{n}+\mathbf{v}}{|\mathbf{n}+\mathbf{v}|} \otimes \frac{\mathbf{n}+\mathbf{v}}{|\mathbf{n}+\mathbf{v}|}=\left(1-\frac{1}{2}|\mathbf{v}|^{2}\right)^{2} \mathbf{n} \otimes \mathbf{n}+\mathbf{n} \otimes \mathbf{v}+\mathbf{v} \otimes \mathbf{n}+\mathbf{v} \otimes \mathbf{v}+o\left(|\mathbf{v}|^{2}\right),
$$

and the first variation of $\boldsymbol{\Theta} \in L^{d-1}$, in the direction $\mathbf{v}$, is

$$
\delta \Theta(\mathbf{v})=\mathbf{n} \otimes \mathbf{v}+\mathbf{v} \otimes \mathbf{n} \in T_{\Theta} L^{d-1}
$$

(the tangent space of $L^{d-1}$ at $\boldsymbol{\Theta}$ ). Thus, at $\boldsymbol{\Theta}=\mathbf{n} \otimes \mathbf{n}$, there is a bijection between $T_{\boldsymbol{\Theta}} L^{d-1}$ and $T_{\mathbf{n}} \mathrm{S}^{d-1}[9]$.

Therefore, let us introduce the space of continuous, piecewise linear, vector-valued functions

$$
\mathbb{V}_{h}=\left\{\mathbf{v}_{h} \in\left[H^{1}(\Omega)\right]^{d}:\left.\mathbf{v}_{h}\right|_{T} \in \mathcal{P}_{1}(T), \forall T \in \mathcal{T}_{h}\right\},
$$

and define the space of (discrete) tangential variations of $\boldsymbol{\Theta}_{h}=\mathbf{n}_{h} \otimes \mathbf{n}_{h}$ by

$$
\begin{gathered}
\mathbb{W}_{h}^{\perp}\left(\mathbf{n}_{h}\right)=\left\{\mathbf{v}_{h} \in \mathbb{V}_{h}: \mathbf{v}_{h}\left(x_{i}\right) \cdot \mathbf{n}_{h}\left(x_{i}\right)=0 \text { for all } x_{i} \in \mathcal{N}_{h}\right\}, \\
\mathbb{W}_{h}^{\perp}\left(\boldsymbol{\Theta}_{h}\right)=\left\{\mathbf{W}_{h} \in \bar{U}_{h}: \mathbf{W}_{h}\left(x_{i}\right)=\mathbf{n}_{h}\left(x_{i}\right) \otimes \mathbf{v}_{h}\left(x_{i}\right)+\mathbf{v}_{h}\left(x_{i}\right) \otimes \mathbf{n}_{h}\left(x_{i}\right),\right. \\
\left.\quad \text { where } \mathbf{v}_{h} \in \mathbb{W}_{h}^{\perp}\left(\mathbf{n}_{h}\right)\right\} .
\end{gathered}
$$

\subsection{Discrete variational derivatives}

Given $k \geq 0$, let $\left(s_{h^{\prime}}^{k} \boldsymbol{\Theta}_{h}^{k}\right) \in \mathcal{A}_{\mathrm{uni}}^{h}\left(g_{h}, \mathbf{M}_{h}\right)$ and we write

$$
s_{i}^{k}:=s_{h}^{k}\left(x_{i}\right), \quad \mathbf{\Theta}_{i}^{k}:=\mathbf{\Theta}_{h}^{k}\left(x_{i}\right), \quad \mathbf{n}_{i}^{k}:=\mathbf{n}_{h}^{k}\left(x_{i}\right), \quad z_{i}:=z_{h}\left(x_{i}\right), \quad \mathbf{v}_{i}:=\mathbf{v}_{h}\left(x_{i}\right) .
$$


The discrete variational derivatives of the energy are given by

$$
\begin{aligned}
& \delta_{\boldsymbol{\Theta}} E_{\text {uni }}^{h}\left[s_{h}^{k}, \Theta_{h}^{k}\right]\left(\mathbf{W}_{h}\right)=\frac{1}{2} \delta_{\boldsymbol{\Theta}} \stackrel{\circ}{\text { uni }} h^{h}\left[s_{h}^{k}, \boldsymbol{\Theta}_{h}^{k}\right]\left(\mathbf{W}_{h}\right), \\
& \delta_{\boldsymbol{\Theta}} \stackrel{\circ}{\mathrm{uni}}_{\mathrm{un}}\left[s_{h}^{k}, \boldsymbol{\Theta}_{h}^{k}\right]\left(\mathbf{W}_{h}\right)=\sum_{i, j=1}^{N} k_{i j}\left(\frac{\left(s_{i}^{k}\right)^{2}+\left(s_{j}^{k}\right)^{2}}{2}\right)\left(\delta_{i j} \boldsymbol{\Theta}_{h}^{k}\right):\left(\delta_{i j} \mathbf{W}_{h}\right),
\end{aligned}
$$

for all $\mathbf{W}_{h} \in \mathbb{W}_{h}^{\perp}\left(\boldsymbol{\Theta}_{h}\right)$, and

$$
\begin{aligned}
& \delta_{s} E_{\text {uni }}^{h}\left[s_{h}^{k}, \Theta_{h}^{k}\right]\left(z_{h}\right)=\frac{d-1}{d}\left(\nabla s_{h}^{k}, \nabla z_{h}\right)+\frac{1}{2} \delta_{s} \stackrel{\circ}{\text { uni }}_{h}\left[s_{h}^{k}, \mathbf{\Theta}_{h}^{k}\right]\left(z_{h}\right)+\frac{1}{\epsilon_{\mathrm{dw}}^{2}}\left(\psi_{\mathrm{LdG}}^{\prime}\left(s_{h}^{k}\right), z_{h}\right), \\
& \delta_{s} \stackrel{\circ}{\mathrm{uni}}_{\mathrm{un}}\left[s_{h}^{k}, \boldsymbol{\Theta}_{h}^{k}\right]\left(z_{h}\right)=\sum_{i, j=1}^{N} k_{i j}\left|\delta_{i j} \boldsymbol{\Theta}_{h}^{k}\right|^{2}\left(\frac{s_{i}^{k} z_{i}+s_{j}^{k} z_{j}}{2}\right),
\end{aligned}
$$

for all $z_{h} \in \mathbb{S}_{h}$. Note that $\delta_{\boldsymbol{\Theta}} \stackrel{\circ}{h}_{\text {uni }}^{h}\left[s_{h}^{k}, \boldsymbol{\Theta}_{h}^{k}\right]\left(\mathbf{W}_{h}\right)$ is linear with respect to $\boldsymbol{\Theta}_{h}^{k}$ and $\mathbf{W}_{h}$ (separately), and $\delta_{s} \stackrel{\circ}{E}_{\text {uni }}\left[s_{h^{\prime}}^{k} \boldsymbol{\Theta}_{h}^{k}\right]\left(z_{h}\right)$ is linear with respect to $s_{h}^{k}$ and $z_{h}$ (separately). As will become clear in the next section, alternating the update of $s_{h}$ and $\boldsymbol{\Theta}_{h}$ yields a convex problem to solve at each step, combined with a simple projection.

\subsection{Minimization algorithms}

The minimization schemes consist of an outer loop within which three stages are performed. The first stage updates $\boldsymbol{\Theta}_{h}$ using one (accelerated) gradient descent step. The second stage projects $\boldsymbol{\Theta}_{h}$ onto the admissible set $\mathcal{A}_{\text {uni }}^{h}\left(g_{h}, \mathbf{M}_{h}\right)$ to enforce the constraint $\boldsymbol{\Theta}_{h}\left(x_{i}\right)=\mathbf{n}_{h}\left(x_{i}\right) \otimes \mathbf{n}_{h}\left(x_{i}\right)$ at the nodes of the mesh. The third stage updates $s_{h}$ using one (accelerated) gradient descent step.

The inner products we use for computing descent directions are as follows. Given a function $\omega \in L^{\infty}(\Omega)$ with $\omega \geq 0$, define the weighted space $H_{\omega}^{1}(\Omega)$ through the norm

$$
\|\mathbf{v}\|_{H_{\omega}^{1}(\Omega)}:=\left(\int_{\Omega}|\mathbf{v}(x)|^{2} d x+\int_{\Omega}|\nabla \mathbf{v}(x)|^{2} \omega(x) d x\right)^{1 / 2},
$$

and write $(\cdot, \cdot)_{H_{\omega}^{1}(\Omega)}$ as its inner product. When updating $\mathbf{n}_{h}$ (equivalently, $\boldsymbol{\Theta}_{h}$ ), we define $a_{\mathbf{n}}^{k}(\mathbf{t}, \mathbf{v})=(\mathbf{t}, \mathbf{v})_{H_{\omega^{k}}^{1}(\Omega)}$, with weight $\omega^{k}=\left(s_{h}^{k}\right)^{2}$. For updating $s_{h}$, we choose $a_{s}(\cdot, \cdot)=(\cdot, \cdot)$, the $L^{2}(\Omega)$ inner product.

In order to obtain a linear system when updating $s_{h}$, we use the following convex splitting technique for $\psi_{\mathrm{LdG}}[52,53,64]$. From (2.3) and (3.3), $\psi_{\mathrm{LdG}}$ has the form $\psi_{\mathrm{LdG}}(s)=$ $K+b_{2} s^{2}-b_{3} s^{3}+b_{4} s^{4}$, where $b_{2} \leq 0$, and $b_{3}, b_{4}>0$. Thus, writing

$$
\psi_{\mathrm{LdG}}(s)=\left[K+D s^{2}\right]-\left[\left(D-b_{2}\right) s^{2}+b_{3} s^{3}-b_{4} s^{4}\right]=: \psi_{c}(s)-\psi_{e}(s),
$$


we have that $\psi_{c}, \psi_{e}$ are convex for all $s \in\left[-\frac{1}{d-1}, 1\right]$ provided $D>0$ is large enough. We then use the following approximation when computing a descent direction:

$$
\left(\psi_{\mathrm{LdG}}^{\prime}\left(s_{h}^{k}+r_{h}^{k}\right), z_{h}\right):=\left(\psi_{c}^{\prime}\left(r_{h}^{k}\right), z_{h}\right)+\left(\psi_{c}^{\prime}\left(s_{h}^{k}\right)-\psi_{e}^{\prime}\left(s_{h}^{k}\right), z_{h}\right),
$$

where the first term on the right is linear in $r_{h}^{k}$. The general scheme we consider is given in Algorithm 5.1.

The standard method in Algorithm 5.1 is equivalent to the method in [9] with $\gamma=\delta t$ (time-step), $\beta_{\mathbf{n}}=\beta_{s}=0, \zeta_{\mathbf{n}}^{k}=\zeta_{s}^{k}=0$ for all $k \geq 0$, and $\alpha_{\mathbf{n}}=\alpha_{s}=1$. The resulting algorithm is monotonically energy decreasing [9, Theorem 2], if $\delta t<C h^{d / 2}$ for some constant $C>0$, i.e.,

$$
E_{\text {uni }}^{h}\left[s_{h}^{N}, \Theta_{h}^{N}\right]+\frac{1}{\delta t}\left(\sum_{k=0}^{N-1}\left\|\mathbf{t}_{h}^{k}\right\|_{H_{\left(s_{h}^{k}\right)^{2}}^{1}(\Omega)}^{2}+\left\|r_{h}^{k}\right\|_{L^{2}(\Omega)}^{2}\right) \leq E_{\text {uni }}^{h}\left[s_{h}^{0}, \Theta_{h}^{0}\right], \quad \forall N \geq 1 .
$$

The reason for the time step restriction is due to the "second order inconsistency" in updating $\boldsymbol{\Theta}_{h}^{k}$ in (5.7). In other words, setting $\widetilde{\boldsymbol{\Theta}}_{h}^{k+1}:=\mathbf{\Theta}_{h}^{k}+\mathbf{T}_{h}^{k}$ it is clear that $\widetilde{\boldsymbol{\Theta}}_{h}^{k+1}\left(x_{i}\right) \neq$ $\mathbf{a} \otimes \mathbf{a} \in L^{d-1}$ for any $\mathbf{a} \in \mathbb{S}^{d-1}$ and any node $x_{i} \in \mathcal{N}_{h}$. According to (5.2), a better option would be to replace $\boldsymbol{\Theta}_{h}^{k}+\mathbf{T}_{h}^{k}$ with

$$
\mathbf{n}_{h}^{k} \otimes \mathbf{n}_{h}^{k}+\mathbf{n}_{h}^{k} \otimes \mathbf{t}_{h}^{k}+\mathbf{t}_{h}^{k} \otimes \mathbf{n}_{h}^{k}+\mathbf{t}_{h}^{k} \otimes \mathbf{t}_{h}^{k} \equiv\left(\mathbf{n}_{h}^{k}+\mathbf{t}_{h}^{k}\right) \otimes\left(\mathbf{n}_{h}^{k}+\mathbf{t}_{h}^{k}\right),
$$

in (5.7), but (5.11) is nonlinear. In Section 5.4, we show that (5.11) can be accounted for by an exact line search with negligible computational cost.

\subsection{Energy decrease and exact line search}

Setting a fixed step size for $\alpha_{\mathbf{n}}$ is simple, but does not guarantee decrease of the energy at each iteration because of the second-order inconsistency mentioned above. Hence, we propose an exact line search. Because of the simple form of $\stackrel{\circ}{E}_{\text {uni }}^{h}\left[s_{h}^{k}, \Theta_{h}^{k}\right]$, we have that

$$
\ell_{\mathbf{n}}(\xi)=\stackrel{\circ}{\mathrm{uni}}_{\text {un }}\left[s_{h}^{k}, \mathcal{I}_{h}\left\{\left(\mathbf{n}_{h}^{k}+\xi_{h}^{k}\right) \otimes\left(\mathbf{n}_{h}^{k}+\xi_{\mathbf{t}_{h}^{k}}^{k}\right)\right\}\right],
$$

is a quartic polynomial in $\xi$. So given $s_{h}^{k} \mathbf{n}_{h}^{k}, \mathbf{t}_{h}^{k}$, we can recover $\ell_{\mathbf{n}}(\xi)$ by sampling at five points. It is then a trivial computation to find the value of $\xi$ that minimizes $\ell_{\mathbf{n}}(\xi)$. Since $E_{\text {uni }}^{h}\left[s_{h}^{k}, \Theta_{h}^{k}\right]$ is convex in $\boldsymbol{\Theta}_{h}^{k}$ (for fixed $s_{h}^{k}$ ), we are guaranteed to find a positive $\xi$ value that minimizes $\ell_{\mathbf{n}}$, unless $\stackrel{\circ}{\text { uni }}_{h}\left[s_{h}^{k} \mathbf{n}_{h}^{k} \otimes \mathbf{n}_{h}^{k}\right]$ is already a minimum; note that

$$
\left(\mathbf{n}_{h}^{k}+\mathbf{t}_{h}^{k}\right) \otimes\left(\mathbf{n}_{h}^{k}+\mathbf{t}_{h}^{k}\right) \approx \boldsymbol{\Theta}_{h}^{k}+\mathbf{T}_{h}^{k}
$$

for $\left|\mathbf{t}_{h}^{k}\right|$ sufficiently small and $\boldsymbol{\Theta}_{h}^{k}+\mathbf{T}_{h}^{k}$ is linear in $\mathbf{t}_{h}^{k}$. Thus, using an exact line search on $\alpha_{\mathbf{n}}>0$ gives

$$
\stackrel{\circ}{E}_{\text {uni }}^{h}\left[s_{h}^{k}, \mathcal{I}_{h}\left\{\left(\mathbf{n}_{h}^{k}+\alpha_{\mathbf{n}} \mathbf{t}_{h}^{k}\right) \otimes\left(\mathbf{n}_{h}^{k}+\alpha_{\mathbf{n}} \mathbf{t}_{h}^{k}\right)\right\}\right]<\stackrel{\circ}{E}_{\text {uni }}^{h}\left[s_{h}^{k}, \mathcal{I}_{h}\left\{\mathbf{n}_{h}^{k} \otimes \mathbf{n}_{h}^{k}\right\}\right],
$$




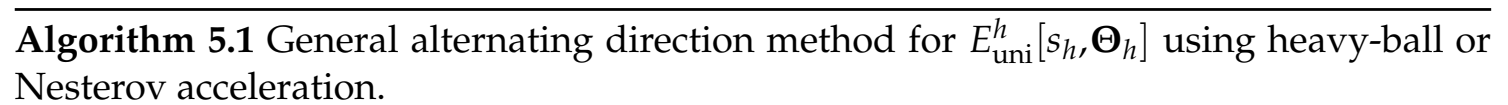

Set a tolerance $\varepsilon_{\text {tol }}>0$ and initial guess $\left(s_{h}^{0}, \boldsymbol{\Theta}_{h}^{0}\right) \in \mathcal{A}_{\text {uni }}^{h}\left(g_{h}, \mathbf{M}_{h}\right)$, with $\boldsymbol{\Theta}_{h}^{0}=I_{h}\left\{\mathbf{n}_{h}^{0} \otimes \mathbf{n}_{h}^{0}\right\}$.

Set $\mathbf{t}_{h}^{-1}=\mathbf{0}, r_{h}^{-1}=0, \mathbf{n}_{h}^{-1}=\mathbf{n}_{h}^{0}, s_{h}^{-1}=s_{h}^{0}$, and set $k:=0$; choose $\gamma_{\mathbf{n}}, \gamma_{s}>0$.

For the standard method: set $\beta_{\mathbf{n}}=\beta_{s}=0$ and $\zeta_{\mathbf{n}}^{k}=\zeta_{s}^{k}=0$ for all $k \geq 0$;

for the heavy-ball method: choose $\beta_{\mathbf{n}}, \beta_{s}>0$ and set $\zeta_{\mathbf{n}}^{k}=\zeta_{s}^{k}=0$ for all $k \geq 0$;

for the Nesterov method: set $\beta_{\mathbf{n}}=\beta_{s}=0$ and update $\zeta_{\mathbf{n}}^{k} \zeta_{s}^{k}>0$ at each iteration using the scheme in

Algorithm 4.2.

For $k \geq 0$, do the following:

1. Tangential search direction for $\mathbf{n}_{h}^{k}$. Compute $\breve{\mathbf{n}}_{h}^{k}=\mathbf{n}_{h}^{k}+\zeta_{\mathbf{n}}^{k}\left(\mathbf{n}_{h}^{k}-\mathbf{n}_{h}^{k-1}\right)$; set $\breve{\Theta}_{h}^{k}=\mathcal{I}_{h}\left\{\breve{\mathbf{n}}_{h}^{k} \otimes \breve{\mathbf{n}}_{h}^{k}\right\} \in \overline{\mathbb{U}}_{h}$. Find $\mathbf{t}_{h}^{k} \in \mathbb{W}_{h}^{\perp}\left(\mathbf{n}_{h}^{k}\right) \cap H_{\Gamma_{\boldsymbol{\Theta}}}^{1}(\Omega)$ and $\mathbf{T}_{h}^{k}=\mathbf{n}_{h}^{k} \otimes \mathbf{t}_{h}^{k}+\mathbf{t}_{h}^{k} \otimes \mathbf{n}_{h}^{k} \in \mathbb{W}_{h}^{\perp}\left(\mathbf{\Theta}_{h}^{k}\right)$, such that

$$
\begin{aligned}
a_{\mathbf{n}}^{k}\left(\mathbf{t}_{h}^{k}, \mathbf{v}_{h}\right)=- & \gamma_{\mathbf{n}} \delta_{\mathbf{\Theta}} \check{E}_{\text {uni }}^{h}\left[s_{h}^{k}, \breve{\Theta}_{h}^{k}+\mathbf{T}_{h}^{k}\right]\left(\mathbf{V}_{h}\right)+\beta_{\mathbf{n}} a_{\mathbf{n}}^{k}\left(\mathbf{t}_{h}^{k-1}, \mathbf{v}_{h}\right), \\
& \forall \mathbf{V}_{h}=\mathbf{n}_{h}^{k} \otimes \mathbf{v}_{h}+\mathbf{v}_{h} \otimes \mathbf{n}_{h}^{k}, \quad \mathbf{v}_{h} \in \mathbb{W}_{h}^{\perp}\left(\mathbf{n}_{h}^{k}\right) \cap H_{\Gamma_{\boldsymbol{\Theta}}}^{1}(\Omega) .
\end{aligned}
$$

2. Update $\mathbf{n}_{h}^{k}$. Let $\tilde{\mathbf{n}}_{h}^{k+1} \in \mathbb{V}_{h}$ with $\tilde{\mathbf{n}}_{h}^{k+1}:=\breve{\mathbf{n}}_{h}^{k}+\alpha_{\mathbf{n}} \mathbf{t}_{h}^{k}$, where $\alpha_{\mathbf{n}}>0$ is the step size (either chosen arbitrarily or found by an exact line-search).

3. Update $\boldsymbol{\Theta}_{h}^{k}$ by projection. Compute $\boldsymbol{\Theta}_{h}^{k+1} \in \mathbb{T}_{h}\left(\Gamma_{\boldsymbol{\Theta}}, \mathbf{M}_{h}\right)$ with

$$
\Theta_{h}^{k+1}\left(x_{i}\right):=\frac{\tilde{\mathbf{n}}_{h}^{k+1}\left(x_{i}\right)}{\left|\tilde{\mathbf{n}}_{h}^{k+1}\left(x_{i}\right)\right|} \otimes \frac{\tilde{\mathbf{n}}_{h}^{k+1}\left(x_{i}\right)}{\left|\tilde{\mathbf{n}}_{h}^{k+1}\left(x_{i}\right)\right|}, \quad \forall x_{i} \in \mathcal{N}_{h} .
$$

4. Search direction for $s_{h}^{k}$. Compute $\breve{s}_{h}^{k}=s_{h}^{k}+\zeta_{s}^{k}\left(s_{h}^{k}-s_{h}^{k-1}\right)$. Find $r_{h}^{k} \in \mathbb{S}_{h}\left(\Gamma_{s}, 0\right)$ such that, for all $z_{h} \in \mathrm{S}_{h}\left(\Gamma_{s}, 0\right)$,

$$
a_{s}\left(r_{h}^{k}, z_{h}\right)=-\gamma_{s} \delta_{s} E_{\text {uni }}^{h}\left[\breve{s}_{h}^{k}+r_{h}^{k}, \Theta_{h}^{k+1}\right]\left(z_{h}\right)+\beta_{s} a_{S}\left(r_{h}^{k-1}, z_{h}\right)
$$

or, more explictly,

$$
\begin{aligned}
& a_{s}\left(r_{h}^{k}, z_{h}\right)+\gamma_{s} \delta_{s} E_{\mathrm{uni}-\mathrm{m}}^{h}\left[r_{h}^{k}, \Theta_{h}^{k+1}\right]\left(z_{h}\right)+\frac{\gamma_{s}}{\epsilon_{\mathrm{dw}}^{2}}\left(\psi_{c}^{\prime}\left(r_{h}^{k}\right), z_{h}\right) \\
= & -\gamma_{s} \delta_{s} E_{\mathrm{uni}-\mathrm{m}}^{h}\left[\breve{s}_{h}^{k}, \Theta_{h}^{k+1}\right]\left(z_{h}\right)-\frac{\gamma_{s}}{\epsilon_{\mathrm{dw}}^{2}}\left(\psi_{\mathrm{LdG}}^{\prime}\left(\breve{s}_{h}^{k}\right), z_{h}\right)+\beta_{s} a_{s}\left(r_{h}^{k-1}, z_{h}\right) .
\end{aligned}
$$

5. Update $s_{h}^{k}$. Let $s_{h}^{k+1} \in \mathbb{S}_{h}\left(\Gamma_{s}, g_{h}\right)$ with $s_{h}^{k+1}:=\breve{s}_{h}^{k}+\alpha_{s} r_{h}^{k}$, where $\alpha_{s}>0$ is the step size (either chosen arbitrarily or found by an exact line-search).

6. If

$$
\left|E_{\text {uni }}^{h}\left[s_{h}^{k+1}, \boldsymbol{\Theta}_{h}^{k+1}\right]-E_{\text {uni }}^{h}\left[s_{h}^{k}, \Theta_{h}^{k}\right]\right|<\varepsilon_{\text {tol }},
$$

then stop; else, replace $k \leftarrow k+1$ and return to Step 1 .

and furthermore, the projection in (5.8) is monotone energy decreasing [6] (because of Assumption 3.1), so that

$$
\stackrel{\circ}{E}_{\text {uni }}^{h}\left[s_{h}^{k}, \boldsymbol{\Theta}_{h}^{k+1}\right] \leq \stackrel{\circ}{E}_{\text {uni }}^{h}\left[s_{h}^{k}, \mathcal{I}_{h}\left\{\left(\mathbf{n}_{h}^{k}+\alpha_{\mathbf{n}} \mathbf{t}_{h}^{k}\right) \otimes\left(\mathbf{n}_{h}^{k}+\alpha_{\mathbf{n}} \mathbf{t}_{h}^{k}\right)\right\}\right]
$$


Moreover, $E_{\text {uni }}^{h}\left[s_{h}^{k+1}, \boldsymbol{\Theta}_{h}^{k+1}\right]$, also has a simple form, i.e., fixing $\mathbf{\Theta}_{h}^{k+1}$,

$$
\ell_{s}(\xi)=E_{\text {uni }}^{h}\left[s_{h}^{k}+\xi r_{h}^{k}, \Theta_{h}^{k+1}\right]
$$

is a quartic polynomial in $\xi$ because of $\psi_{\mathrm{LdG}}$. Hence, we can easily do an exact line search when updating $s_{h}^{k}$ as above. In the next section, we show the efficacy of this approach.

\section{Numerical experiments}

We tested the three schemes: standard method, heavy-ball method, Nesterov method given in Algorithm 5.1, by simulating a point defect in two dimensions (Section 6.1) and line defect in three dimensions (Section 6.3). Simulations were performed for a sequence of values for $\epsilon_{\mathrm{dw}}$. A sequence of mesh sizes $h_{D}$ was also used, but only the finest mesh is shown here (the performance trends were similar for the other mesh sizes). The stopping criteria for all cases is

$$
\left|E_{\text {uni }}^{h}\left[s_{h}^{k+1}, \Theta_{h}^{k+1}\right]-E_{\text {uni }}^{h}\left[s_{h}^{k}, \Theta_{h}^{k}\right]\right|<\varepsilon_{\text {tol }}:=10^{-6} .
$$

The schemes were implemented using the MATLAB/C++ finite element toolbox FELICITY [60]. In the 3-D simulations, the linear system solves were done using the algebraic multi-grid solver-AGMG [38,39]. The computations were carried out on a Dell XPS desktop, 6 core Intel i7 3.20GHz with 64GB RAM.

\subsection{Experiment 1: point defect in 2D}

\subsubsection{Simulation statistics}

For the uniaxially constrained LdG model, we use the following double well potential $\psi_{\mathrm{LdG}}(s)$ :

$$
\psi_{\mathrm{LdG}}(s)=\psi_{c}(s)-\psi_{e}(s)=1-8.16325 s^{2}+33.31945 s^{4},
$$

such that

$$
\psi_{c}(s)=K+\frac{(A+D)}{2} s^{2}+\frac{C}{4} s^{4} \quad \text { and } \quad \psi_{e}(s)=\frac{D}{2} s^{2}-\frac{C}{4} s^{4}
$$

with $A=-16.3265, C=66.6389, D=144.040024$, and $K=1.0$. Note this splitting is only convex for $-1 \leq s \leq+1$ (all simulations given here satisfy these bounds).

The Dirichlet boundary conditions imposed on the liquid crystal domain are

$$
\begin{array}{ll}
s=s^{*}=0.7 & \text { on } \Gamma_{s}, \\
\mathbf{n}(x, y)=[\cos (\theta), \sin (\theta)]^{T}, \quad \theta=\operatorname{atan} 2\left(\frac{y-y_{0}}{x-x_{0}}\right) & \text { on } \Gamma_{\boldsymbol{\Theta}},
\end{array}
$$




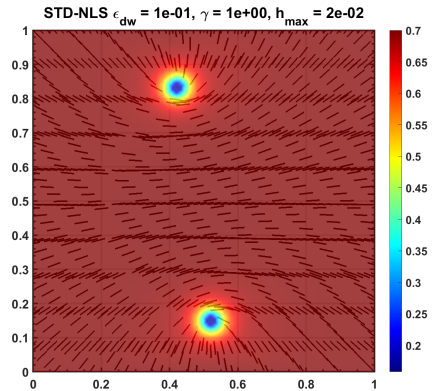

(a) Standard gradient descent algorithm

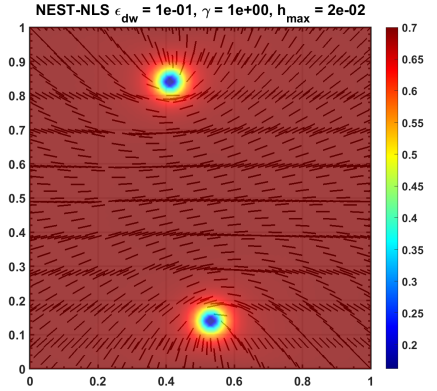

(b) Nesterov accelerated gradient descent algorithm

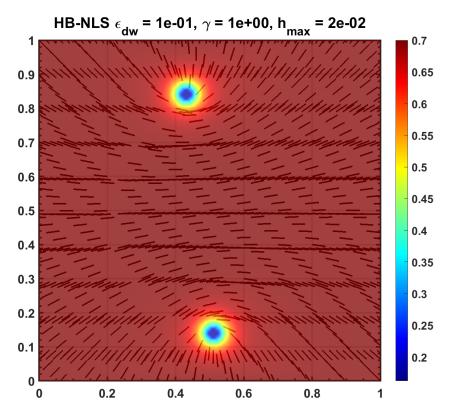

(c) Heavy-Ball accelerated gradient descent algorithm

Figure 1: Point defect in 2D. Equilibrium solution of each algorithm without using an exact line search: for $\epsilon_{\mathrm{dw}}=0.1, \gamma=1, \alpha_{\mathbf{n}}=\alpha_{s}=1$, and $h_{D}=0.02$. Line segments depict $\mathbf{n}$ without an arrowhead; color represents the variable $s$.

where $\boldsymbol{\Theta}=\mathbf{n} \otimes \mathbf{n}$ is defined on $\Gamma_{s}, \Gamma_{\boldsymbol{\Theta}}=\partial \Omega$ and $\left[x_{0}, y_{0}\right]^{T}=[0.5,0.5]^{T}$. The minimization schemes were initialized with

$$
\begin{aligned}
& s_{0}=s^{*}=0.7, \quad \mathbf{n}_{0}(x, y)=\left[\cos \left(\theta_{0}\right), \sin \left(\theta_{0}\right)\right]^{T}, \quad \theta_{0}=\operatorname{atan} 2\left(\frac{y-y_{0}}{x-x_{0}}\right), \\
& {\left[x_{0}, y_{0}\right]^{T}=[0.182,0.31]^{T}, \quad \boldsymbol{\Theta}_{0}=\mathbf{n}_{0} \otimes \mathbf{n}_{0} .}
\end{aligned}
$$

Fig. 1 and Fig. 2 shows the energy minimizer using the three gradient descent minimization schemes without and with exact line search method, respectively. The boundary conditions for $\boldsymbol{\Theta}$ here correspond to a +1 degree point defect, as well as for the initial condition. The initial +1 degree defect splits into two $+1 / 2$ defects because this more easily reduces the elastic energy. All three schemes capture this phenomena exactly.

The energy of the minimizer using all three schemes, as a function of the liquid crystal material property $\epsilon_{\mathrm{dw}}$, is tabulated in Table 1 and Table 2 for each minimization scheme without exact line search and with exact line search, respectively. The relative error in the energy of the minimizer between the minimization schemes is shown in Fig. 3 and Fig. 4 


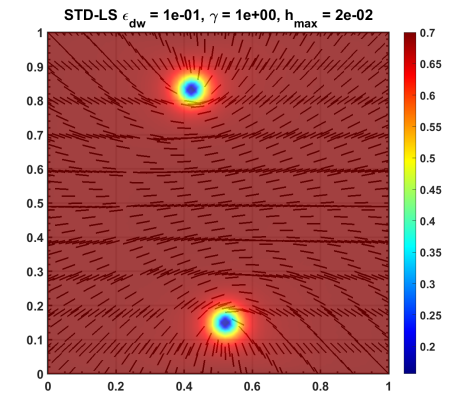

(a) Standard gradient descent algorithm

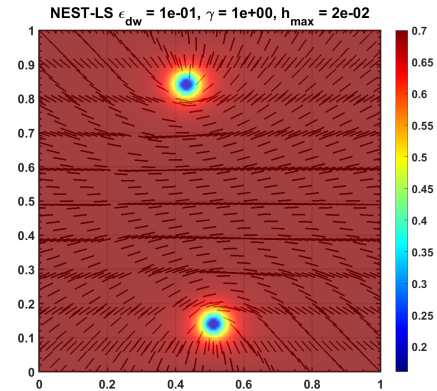

(b) Nesterov accelerated gradient descent algorithm

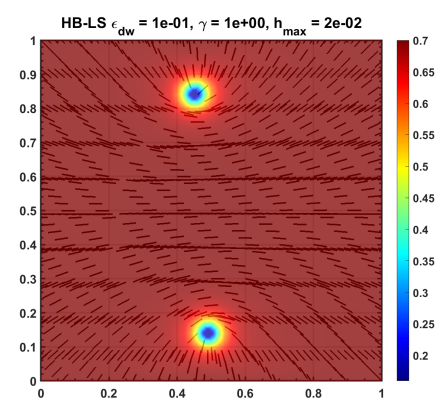

(c) Heavy-Ball accelerated gradient descent algorithm

Figure 2: Point defect in 2D. Equilibrium solution of each algorithm with using an exact line search: for $\epsilon_{\mathrm{dw}}=0.1, \gamma=1$, and $h_{D}=0.02$. Line segments depict $\mathbf{n}$ without an arrowhead; color represents the variable $s$.

Table 1: Equilibrium energy (without line search): $\gamma=1, h_{D}=0.02, \alpha_{\mathbf{n}}=\alpha_{s}=1$. The energy is almost constant across each row, meaning the solution from each of the methods is essentially the same.

\begin{tabular}{||c|c|c|c||}
\hline$\epsilon_{\mathrm{dw}}$ & Standard & Heavy-Ball & Nesterov \\
\hline 0.5 & 2.9241 & 2.9244 & 2.9255 \\
\hline 0.4 & 3.2300 & 3.2244 & 3.2281 \\
\hline 0.2 & 4.2571 & 4.2522 & 4.2571 \\
\hline 0.1 & 5.3184 & 5.3122 & 5.3133 \\
\hline
\end{tabular}

without and with using an exact line search, respectively.

\subsubsection{Comparison of the heavy-ball and Nesterov accelerated gradient descent schemes}

The performance of the accelerated gradient descent schemes - heavy-ball (HB) and Nesterov (NEST) - were compared to the standard gradient descent scheme [9]. The performance of the schemes with and without an exact line search is quantitatively measured in terms of the number of iterations and overall computational time. The implementation of the line search is labeled with LS while without the line search is labeled with NLS. 


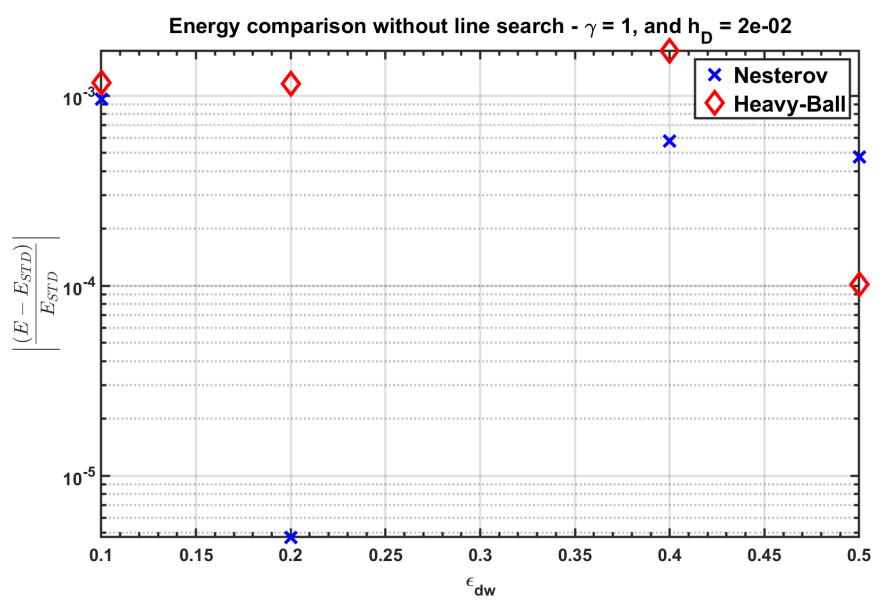

Figure 3: Comparison of the minimum energy for each of the accelerated gradient descent schemes with respect to the "standard" scheme without using exact line search $-\gamma=1, \alpha_{\mathbf{n}}=\alpha_{s}=1$, and $h_{D}=0.02$.

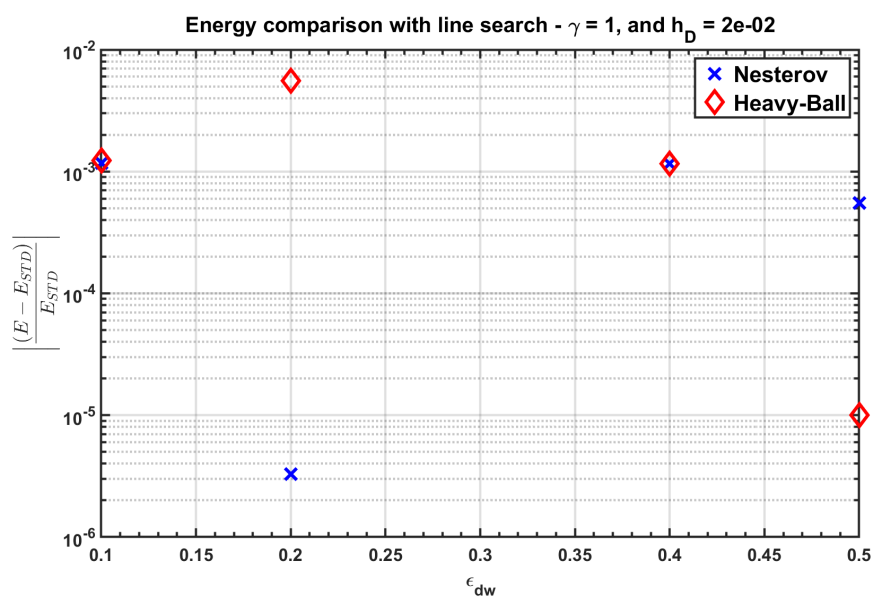

Figure 4: Comparison of the minimum energy for each of the accelerated gradient descent schemes with respect to the "standard" scheme with using exact line search $-\gamma=1$ and $h_{D}=0.02$.

Table 2: Equilibrium energy (with line search): $\gamma=1, h_{D}=0.02$. The energy is almost constant across each row, meaning the solution from each of the methods is essentially the same.

\begin{tabular}{||c|c|c|c||}
\hline$\epsilon_{\mathrm{dw}}$ & Standard & Heavy-Ball & Nesterov \\
\hline 0.5 & 2.9236 & 2.9236 & 2.9252 \\
\hline 0.4 & 3.2281 & 3.2244 & 3.2243 \\
\hline 0.2 & 4.2571 & 4.2335 & 4.2571 \\
\hline 0.1 & 5.3184 & 5.3118 & 5.3122 \\
\hline
\end{tabular}




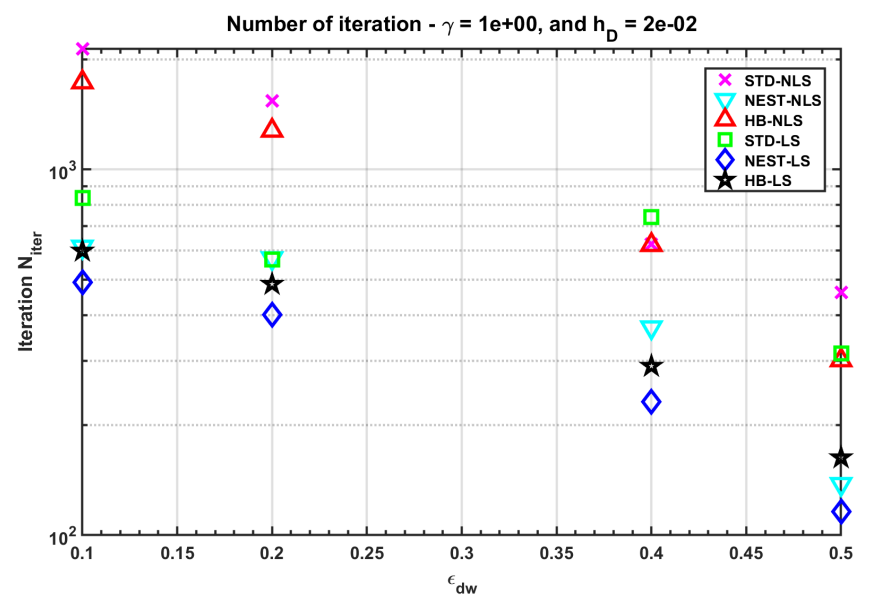

Figure 5: Comparison of the number of iterations required to attain the minimizer using the various minimization schemes.

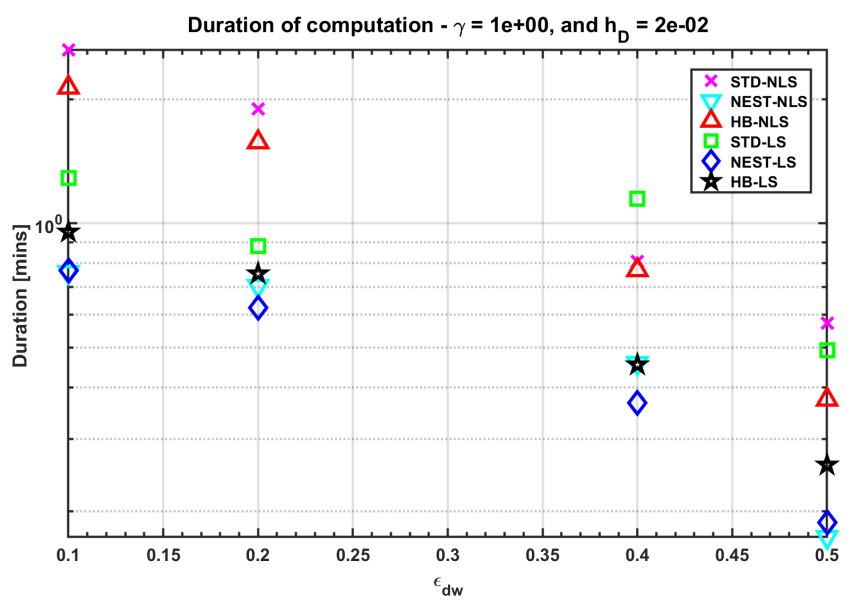

Figure 6: Comparison of the computational time required to attain the minimizer using the various minimization schemes.

Fig. 5 shows the number of iterations needed for the computation to find a minimizer. As shown, the Nesterov method required the fewest iterations in comparison to the other methods (with or without line search). In fact, both accelerated schemes dramatically reduce the number of iterations. For example, the reduction from STD-NLS to NEST-NLS is more than a factor of 3.0. The use of an exact line search improves the performance of all methods.

Moreover, the computational (wall) time was reduced when using the accelerated gradient descent schemes; see Fig. 6 for the total computational time for all of the schemes. For example, the reduction from STD-NLS to NEST-NLS is more than a factor of 3.0. 
Overall, NEST performed best.

\subsection{Experiment 2: point defect in 2D with finer mesh and smaller $\epsilon_{\mathrm{dw}}$}

\subsubsection{Simulation statistics}

The setup of this problem is almost exactly the same as in Section 6.1.1, except for the following. A finer mesh was used $\left(h_{D}=5 \times 10^{-3}\right)$, we take $\epsilon_{\mathrm{dw}}$ down to 0.02 , and the initial coordinates of the degree +1 , point defect are $\left[x_{0}, y_{0}\right]^{T}=[0.762,0.912]^{T}$.

Fig. 7 and Fig. 8 shows the energy minimizer using the three gradient descent minimization schemes without and with exact line search method, respectively. The boundary conditions for $\boldsymbol{\Theta}$ here correspond to a +1 degree point defect, as well as for the initial condition. The initial +1 degree defect splits into two $+1 / 2$ defects because this more easily reduces the elastic energy. All three schemes capture this phenomena exactly. The positioning of the defects in Fig. 7 and Fig. 8 was essentially the same for all values of $\epsilon_{\mathrm{dw}}$ we simulated. Note that the equilibrium configuration found here is different than

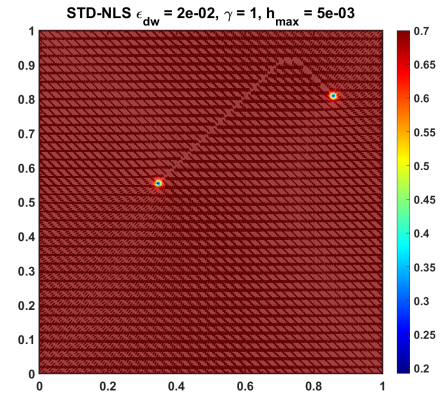

(a) Standard gradient descent algorithm

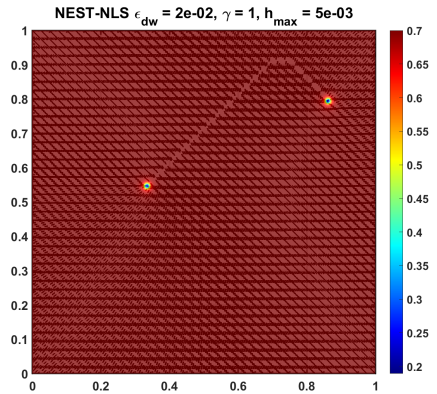

(b) Nesterov accelerated gradient descent algorithm

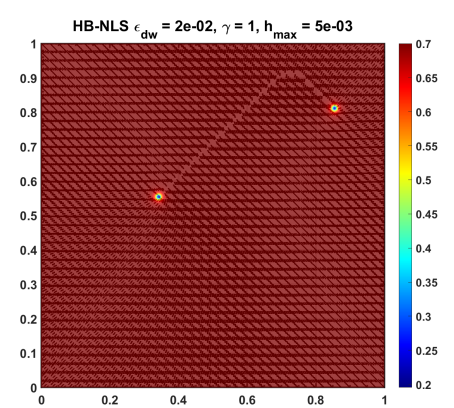

(c) Heavy-Ball accelerated gradient descent algorithm

Figure 7: Point defect in 2D. Equilibrium solution of each algorithm without using an exact line search: for $\epsilon_{\mathrm{dw}}=2 \times 10^{-2}, \gamma=1, \alpha_{\mathbf{n}}=\alpha_{s}=1$, and $h_{D}=5 \times 10^{-3}$. Line segments depict $\mathbf{n}$ without an arrowhead; color represents the variable $s$. Note: the apparent "scar" in the line field plot is just a plotting artifact of Matlab's quiver command. 


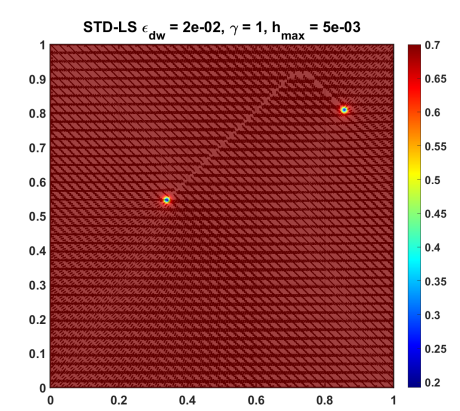

(a) Standard gradient descent algorithm

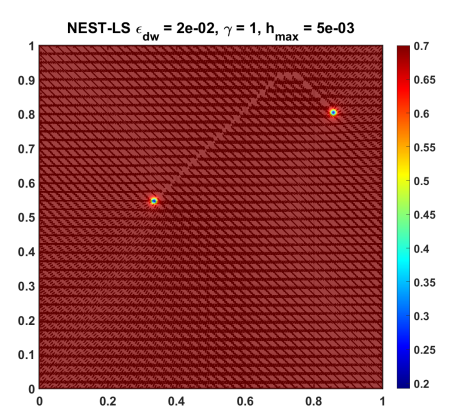

(b) Nesterov accelerated gradient descent algorithm

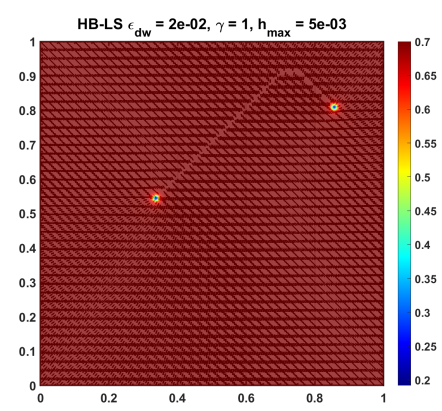

(c) Heavy-Ball accelerated gradient descent algorithm

Figure 8: Point defect in 2D. Equilibrium solution of each algorithm with using an exact line search: for $\epsilon_{\mathrm{dw}}=2 \times 10^{-2}, \gamma=1$, and $h_{D}=5 \times 10^{-3}$. Line segments depict $\mathbf{n}$ without an arrowhead; color represents the variable $s$. Note: the apparent "scar" in the line field plot is just a plotting artifact of Matlab's quiver command.

the one in Section 6.1.1. This is due to the fact that the energy functional $E_{\text {uni }}[s, \boldsymbol{\Theta}]$ is not convex and we used a different initial condition for the point defect.

The energy of the minimizer using all three schemes, as a function of the liquid crystal material property $\epsilon_{\mathrm{dw}}$, is tabulated in Table 3 and Table 4 for each minimization scheme without exact line search and with exact line search, respectively. The relative error in the energy of the minimizer between the minimization schemes is shown in Fig. 9 and

Table 3: Equilibrium energy (without line search): $\gamma=1, h_{D}=5 \times 10^{-3}, \alpha_{\mathbf{n}}=\alpha_{s}=1$. The energy is almost constant across each row, meaning the solution from each of the methods is essentially the same.

\begin{tabular}{||c|c|c|c||}
\hline$\epsilon_{\mathrm{dw}}$ & Standard & Heavy-Ball & Nesterov \\
\hline 0.2 & 4.2353 & 4.2367 & 4.2387 \\
\hline 0.1 & 5.3213 & 5.3215 & 5.3263 \\
\hline 0.05 & 6.4362 & 6.4281 & 6.4327 \\
\hline 0.02 & 8.7118 & 8.6976 & 8.6135 \\
\hline
\end{tabular}


Table 4: Equilibrium energy (with line search): $\gamma=1, h_{D}=5 \times 10^{-3}$. The energy is almost constant across each row, meaning the solution from each of the methods is essentially the same.

\begin{tabular}{||c|c|c|c||}
\hline$\epsilon_{\mathrm{dw}}$ & Standard & Heavy-Ball & Nesterov \\
\hline 0.2 & 4.2314 & 4.2367 & 4.2338 \\
\hline 0.1 & 5.3235 & 5.3223 & 5.3222 \\
\hline 0.05 & 6.5324 & 6.4230 & 6.4303 \\
\hline 0.02 & 8.6669 & 8.6522 & 8.6342 \\
\hline
\end{tabular}

Fig. 10 without and with using an exact line search, respectively.

\subsubsection{Comparison of the heavy-ball and Nesterov accelerated gradient descent schemes}

The performance of the accelerated gradient descent schemes-heavy-ball (HB) and Nesterov (NEST)-were compared to the standard gradient descent scheme [9]. The performance of the schemes with and without an exact line search is quantitatively measured in terms of the number of iterations and overall computational time. The implementation of the line search is labeled with LS while without the line search is labeled with NLS.

Fig. 11 shows the number of iterations needed for the computation to find a minimizer. As shown, the Nesterov method required the fewest iterations, for the smaller values of $\epsilon_{\mathrm{dw}}$, in comparison to the other methods (with or without line search). In fact, both accelerated schemes dramatically reduce the number of iterations. For example, the reduction from STD-NLS to NEST-NLS is more than a factor of 3.0 (for smaller values of $\left.\epsilon_{\mathrm{dw}}\right)$. The use of an exact line search improves the performance of all methods.

Moreover, the computational (wall) time was reduced when using the accelerated gra-

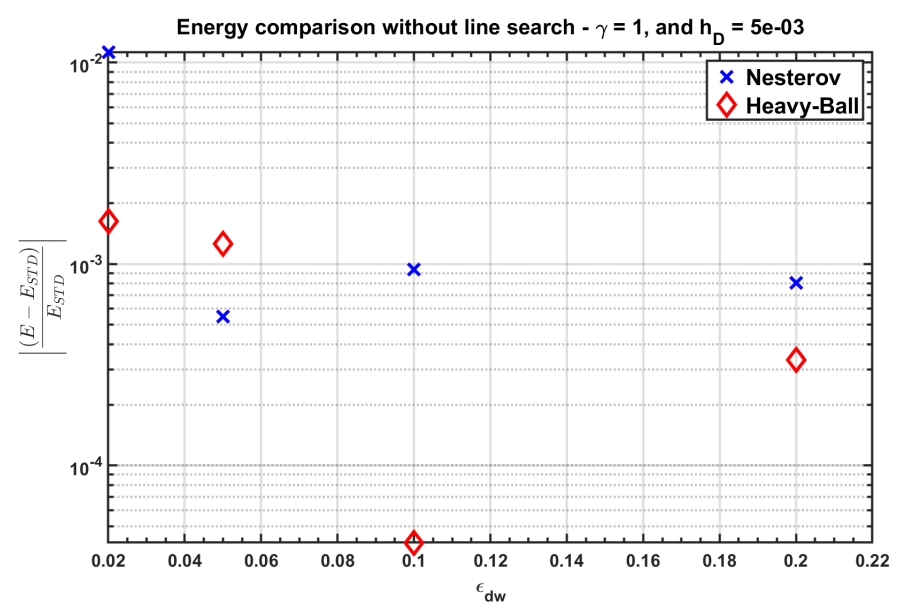

Figure 9: Comparison of the minimum energy for each of the accelerated gradient descent schemes with respect to the "standard" scheme without using exact line search $-\gamma=1, \alpha_{\mathbf{n}}=\alpha_{s}=1$, and $h_{D}=5 \times 10^{-3}$. 


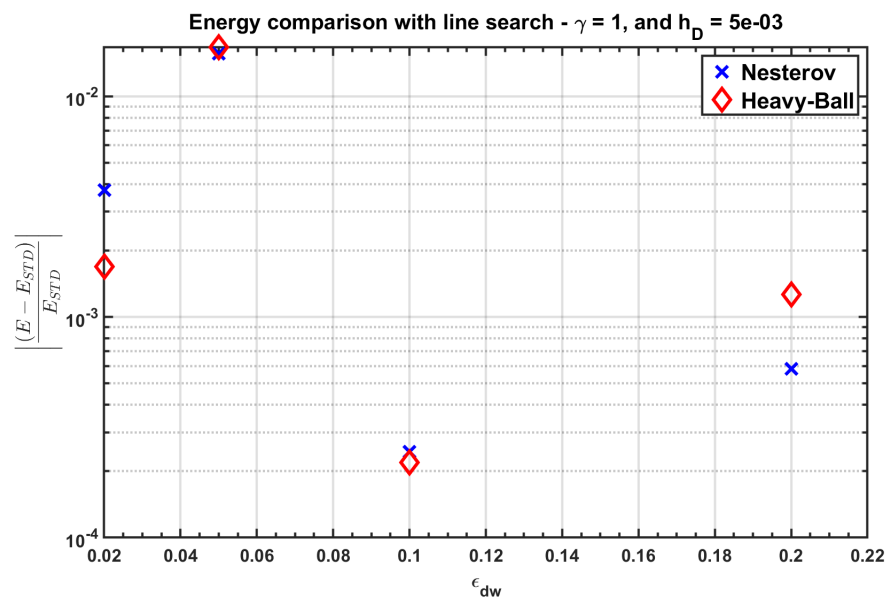

Figure 10: Comparison of the minimum energy for each of the accelerated gradient descent schemes with respect to the "standard" scheme with using exact line search $-\gamma=1$ and $h_{D}=5 \times 10^{-3}$.

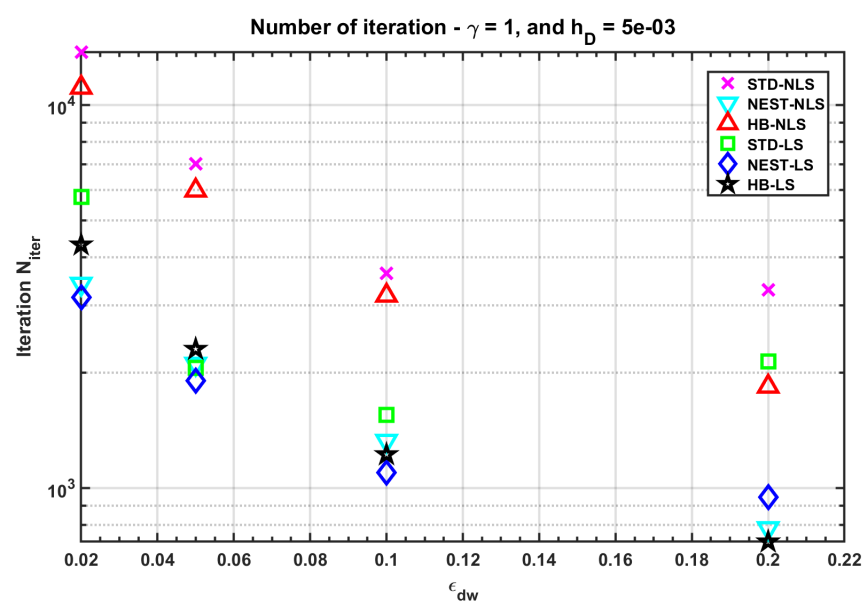

Figure 11: Comparison of the number of iterations required to attain the minimizer using the various minimization schemes.

dient descent schemes; see Fig. 12 for the total computational time for all of the schemes. For example, the reduction from STD-NLS to NEST-NLS is more than a factor of 3.0. Overall, NEST performed best.

\subsection{Experiment 3: line defect in 3D}




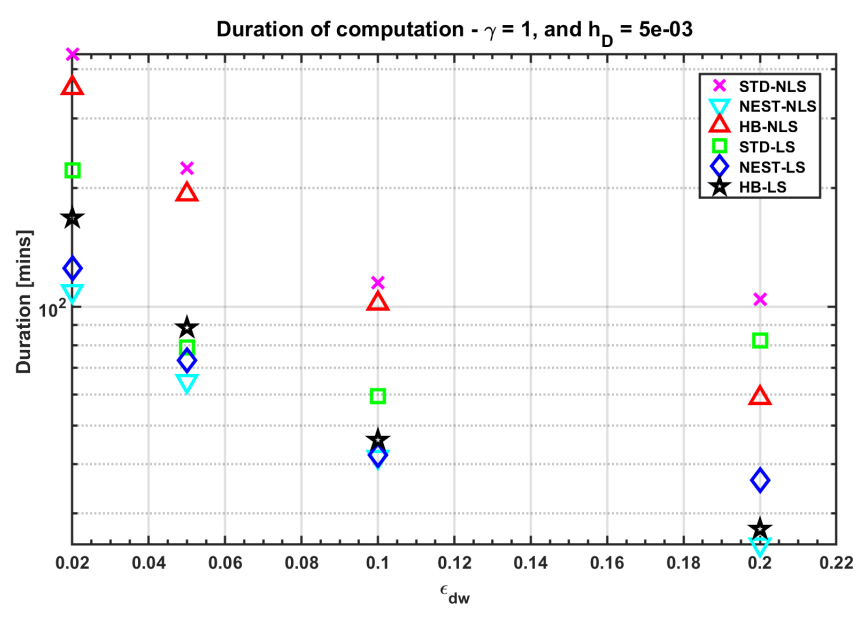

Figure 12: Comparison of the computational time required to attain the minimizer using the various minimization schemes.

\subsubsection{Simulation statistics}

For the uniaxially constrained LdG model, we use the following double well potential $\psi_{\mathrm{LdG}}(s)$ :

$$
\psi_{\mathrm{LdG}}(s)=\psi_{c}(s)-\psi_{e}(s)=1-3.75104 s^{2}-40.6504 s^{3}+33.259425 s^{4},
$$

such that

$$
\psi_{c}(s)=K+\frac{(A+D)}{2} s^{2}-\frac{B}{3} s^{3}+\frac{C}{4} s^{4} \quad \text { and } \quad \psi_{e}(s)=\frac{D}{2} s^{2}+\frac{B}{3} s^{3}-\frac{C}{4} s^{4}
$$

with $A=-7.50208, B=60.9756, C=66.51885, D=552.22912$, and $K=1.0$. Note this splitting is only convex for $-1 / 2 \leq s \leq+1$ (all simulations given here satisfy these bounds).

The domain $\Omega$ has a hole cut out such that its center is located at $\left(x_{c}, y_{c}, z_{c}\right)$ and has a radius $R_{\text {hole }}$. The Dirichlet boundary conditions imposed on the liquid crystal domain is

$$
\begin{aligned}
& s=s^{*}=0.7 \quad \text { on } \Gamma_{s}=\partial \Omega, \\
& \mathbf{n}(x, y, z)=\left\{\begin{array}{lll}
\frac{\mathbf{n}_{b c}}{\left|\mathbf{n}_{b c}\right|} \text { on } \Gamma_{\text {hole }}, \\
{[0,0,1]^{T} \text { on } \Gamma_{\boldsymbol{\Theta}}=\partial \Omega \backslash \Gamma_{\text {hole, }}}
\end{array}\right. \\
& \mathbf{\Theta}=\mathbf{n} \otimes \mathbf{n} \text { is defined on } \Gamma_{s}, \quad \Gamma_{\boldsymbol{\Theta}}=\partial \Omega,
\end{aligned}
$$

where $\mathbf{n}_{b c}=\left[x-x_{c}, y-y_{c}, z-z_{c}\right]^{T}$, and $\left[x_{c}, y_{c}, z_{c}\right]^{T}=[0.35355,0.35355,0]^{T}, R_{\text {hole }}=0.20011$. The minimization schemes were initialized with $s_{0}=s^{*}=0.7, \mathbf{n}_{0}(x, y)=[0,0,1]^{T}$, and $\boldsymbol{\Theta}_{0}=$ $\mathbf{n}_{0} \otimes \mathbf{n}_{0}$ on $\Omega$.

Fig. 13 and Fig. 14 shows the energy minimizer using the three gradient descent minimization schemes without and with exact line search method, respectively. A defect 


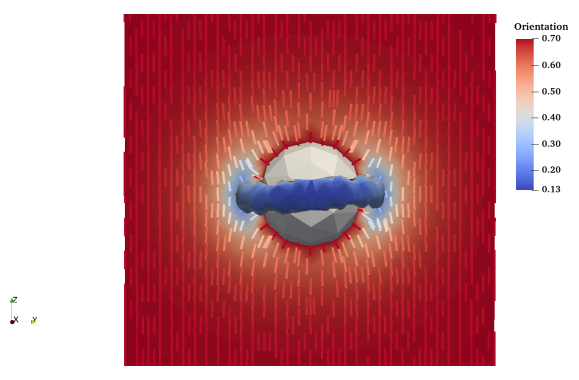

(a) Standard gradient descent

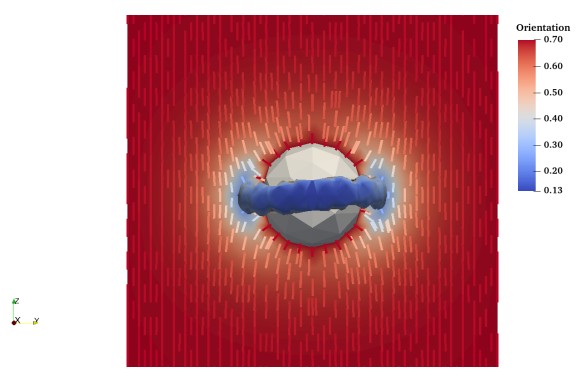

(b) Nesterov accelerated gradient descent

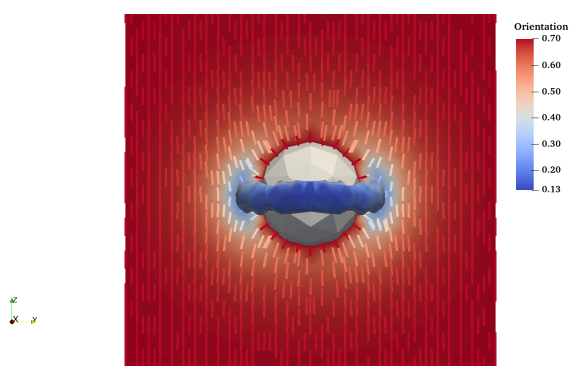

(c) Heavy-Ball accelerated gradient descent

Figure 13: Saturn-ring defect in 3D (vertical slice shown). Equilibrium solution of each algorithm without using an exact line search: for $\epsilon_{\mathrm{dw}}=0.2, \gamma=1, \alpha_{\mathbf{n}}=\alpha_{s}=1$, and $h_{D}=0.06$. Line segments depict $\mathbf{n}$ without an arrowhead; color represents the variable $s$. The ring defect is denoted by the $s=0.25$ iso-surface (light blue).

Table 5: Equilibrium energy (without line search): $\gamma=1, h_{D}=0.06, \alpha_{\mathbf{n}}=\alpha_{s}=1$. The energy is almost constant across each row, meaning the solution from each of the methods is essentially the same.

\begin{tabular}{||c|c|c|c||}
\hline$\epsilon_{\mathrm{dw}}$ & STD & Heavy-Ball & Nesterov \\
\hline 0.5 & 2.4195 & 2.4193 & 2.4195 \\
\hline 0.4 & 2.5360 & 2.5360 & 2.5360 \\
\hline 0.3 & 2.7016 & 2.7016 & 2.7016 \\
\hline 0.2 & 2.9572 & 2.9572 & 2.9572 \\
\hline
\end{tabular}

Table 6: Equilibrium energy (with line search): $\gamma=1, h_{D}=0.06$. The energy is almost constant across each row, meaning the solution from each of the methods is essentially the same.

\begin{tabular}{||c|c|c|c||}
\hline$\epsilon_{\mathrm{dw}}$ & STD & Heavy-Ball & Nesterov \\
\hline 0.5 & 2.4195 & 2.4196 & 2.4195 \\
\hline 0.4 & 2.5360 & 2.5360 & 2.5360 \\
\hline 0.3 & 2.7016 & 2.7016 & 2.7016 \\
\hline 0.2 & 2.9572 & 2.9572 & 2.9572 \\
\hline
\end{tabular}

region in the shape of a ring around the equator of the hole develops, which is observed in experiments [21]; see also [3]. All three schemes capture this phenomena exactly. 


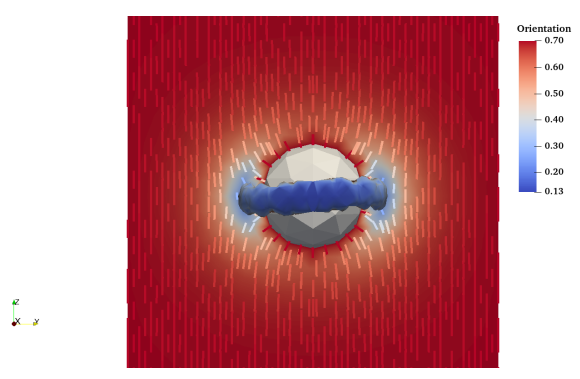

(a) Standard gradient descent algorithm

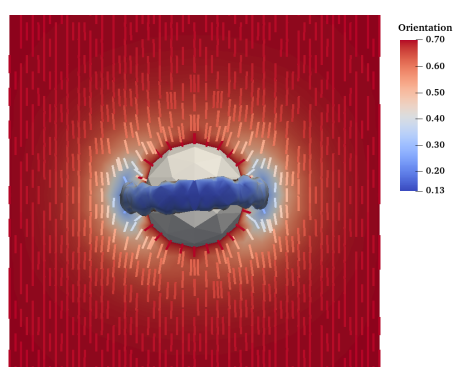

(b) Nesterov accelerated gradient descent algorithm

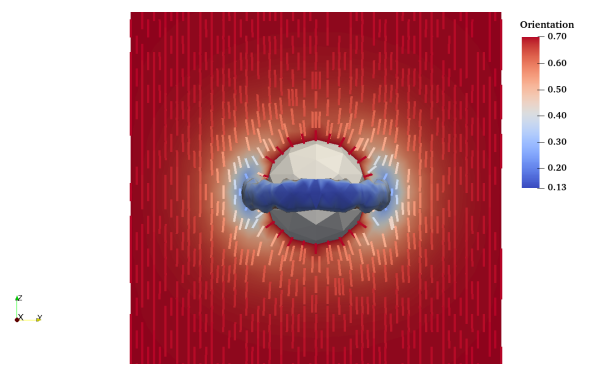

(c) Heavy-Ball accelerated gradient descent algorithm

Figure 14: Saturn-ring defect in 3D (vertical slice shown). Equilibrium solution of each algorithm with using an exact line search: for $\epsilon_{\mathrm{dw}}=0.2, \gamma=1$, and $h_{D}=0.06$. Line segments depict $\mathbf{n}$ without an arrowhead; color represents the variable $s$. The ring defect is denoted by the $s=0.25$ iso-surface (light blue).

The energy of the minimizer using all three schemes, as a function of the liquid crystal material property $\epsilon_{\mathrm{dw}}$, is tabulated in Table 5 and Table 6 for each minimization scheme without exact line search and with exact line search, respectively. The relative error in the energy of the minimizer between the minimization schemes is shown in Fig. 15 and Fig. 16 without and with using an exact line search, respectively.

\subsubsection{Comparison of the heavy-ball and Nesterov accelerated gradient descent schemes}

The performance of the accelerated gradient descent schemes-heavy-ball (HB) and Nesterov (NEST)-were compared to the standard gradient descent scheme [9]. The performance of the schemes with and without an exact line search is quantitatively measured in terms of the number of iterations and overall computational time. The implementation of the line search is labeled with LS while without the line search is labeled with NLS.

Fig. 17 shows the number of iterations needed for the computation to find a minimizer. As shown, the Nesterov method required the fewest iterations in comparison to the other methods (with or without line search). In fact, both accelerated schemes dramatically reduce the number of iterations. For example, the reduction from STD-NLS to NEST-NLS is more than a factor of 2.0. The use of an exact line search improves the 


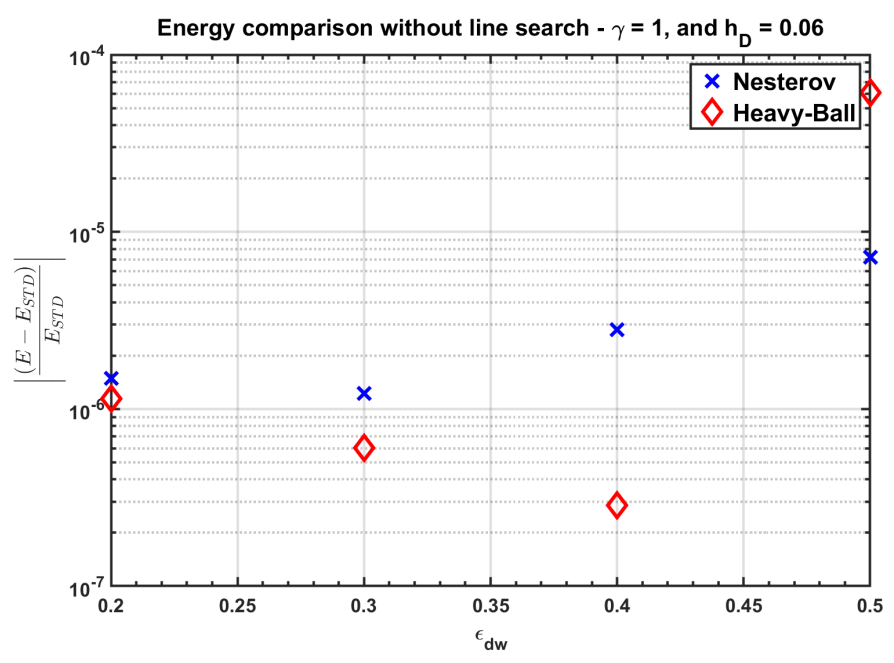

Figure 15: Comparison of the minimum energy for each of the accelerated gradient descent schemes with respect to the "standard" scheme without using exact line search $-\gamma=1, \alpha_{\mathbf{n}}=\alpha_{s}=1$, and $h_{D}=0.06$.

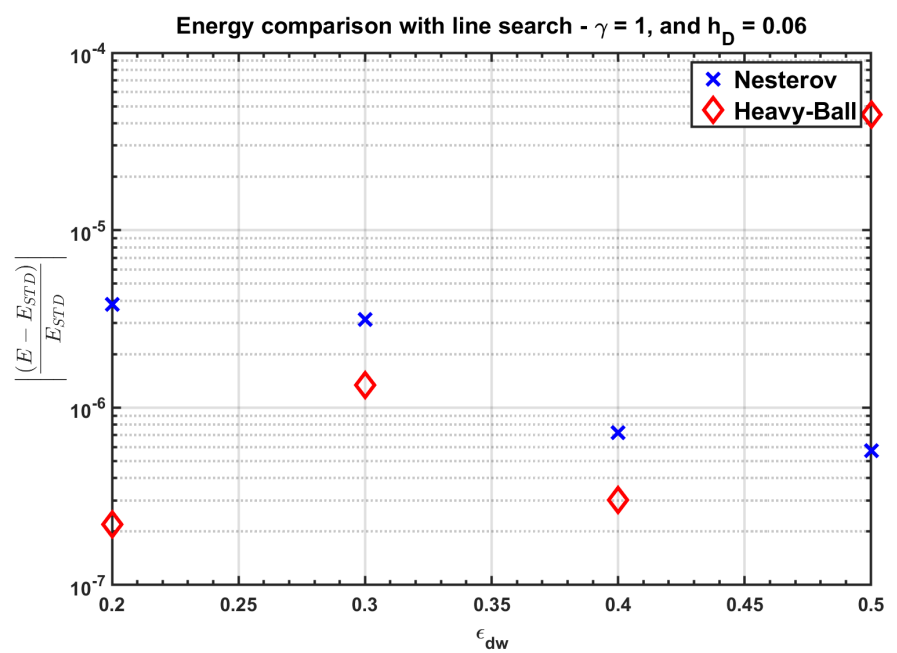

Figure 16: Comparison of the minimum energy for each of the accelerated gradient descent schemes with respect to the "standard" scheme with using exact line search $-\gamma=1$ and $h_{D}=0.06$.

performance of all methods.

Moreover, the computational (wall) time was reduced when using the accelerated gradient descent schemes; see Fig. 18 for the total computational time for all of the schemes. For example, the reduction from STD-NLS to NEST-NLS is about a factor of 2.5. Overall, NEST performed best. 


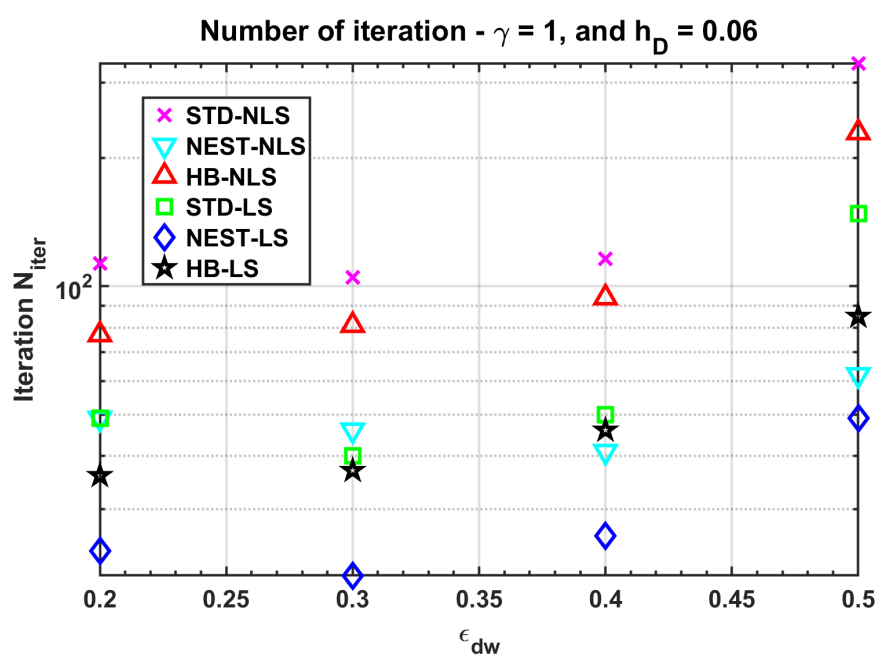

Figure 17: Comparison of the number of iterations required to attain the minimizer using the various minimization schemes.

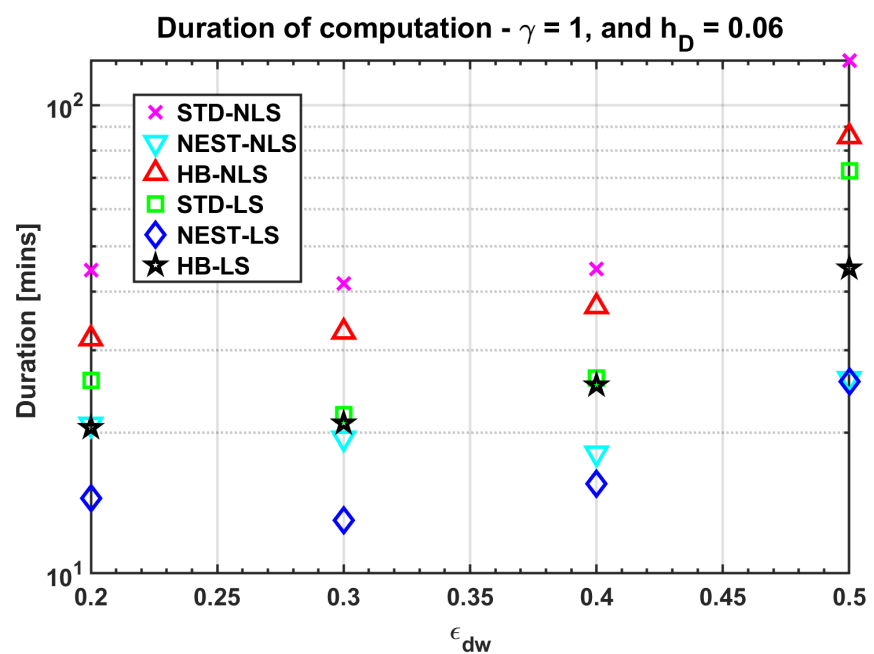

Figure 18: Comparison of the computational time required to attain the minimizer using the various minimization schemes.

\section{Conclusions}

We considered a unique model of liquid crystals that enforces a uniaxial constraint on the Landau-de Gennes model (Section 3). Moreover, we presented numerical experiments demonstrating that accelerated gradient descent schemes, i.e., the heavy-ball method and Nesterov method, significantly speed up simulations for computing minimizers of this 
model when compared to a more standard gradient descent method. In addition, using an exact line search, which is not expensive for the uniaxial model, also greatly reduces the computational effort.

It is known, both computationally and theoretically, that the heavy-ball method and Nesterov scheme work well for many types of optimization problems. What is surprising here is that these schemes still work very well despite the uniaxial constraint, which is not smooth when defects are present. It is well-known in the modeling of LCs that defects have slow dynamics under gradient flows, especially when $\epsilon_{\mathrm{dw}}>0$ is very small. Furthermore, the alternating direction minimization can further exacerbate the slowness of defects, as described in [12].

On the other hand, the alternating scheme is very robust and relatively easy to implement. Therefore, there are two contributions of this paper: (i) to give direct evidence that accelerated gradient descent schemes can augment the alternating scheme and make it more efficient, without much extra complexity in implementation; and (ii) accelerated gradient schemes can overcome "hard" constraints, such as uniaxiality.

\section{Acknowledgements}

The second author (Prof. S. W. Walker) was supported in part by NSF grant DMS-1555222 (CAREER).

\section{References}

[1] Bharat R. Acharya, Andrew Primak, AND SATYEndra KumAR, Biaxial nematic phase in bent-core thermotropic mesogens, Phys. Rev. Lett., 92 (2004), 145506.

[2] ÁGNEs BuKA AND NÁNDOR ÉBER, Flexoelectricity in Liquid Crystals: Theory, Experiments and Applications, World Scientific, 2012.

[3] Stan Alama, Lia Bronsard, AND XaVier LAMY, Analytical description of the saturn-ring defect in nematic colloids, Phys. Rev. E, 93 (2016), 012705.

[4] IVO BABUŠKA AND A. K. AZIZ, On the angle condition in the finite element method, SIAM J. Numer. Anal., 13(2) (1976), pp. 214-226.

[5] IZTOK BAJC, FrÉDÉRIC HECHT, AND SLOBODAn ŽUMER, A mesh adaptivity scheme on the landau-de gennes functional minimization case in $3 d$, and its driving efficiency, J. Comput. Phys., 321 (2016), pp. 981-996.

[6] SÖREn BARTEls AND AlexANDER RAISCH, Simulation of Q-tensor fields with constant orientational order parameter in the theory of uniaxial nematic liquid crystals, in Michael Griebel, editor, Singular Phenomena and Scaling in Mathematical Models, pages 383-412, Springer International Publishing, 2014.

[7] J. S. BIGgins, M. WARNER, AND K. BHATTACHARYA, Elasticity of polydomain liquid crystal elastomers, J. Mech. Phys. Solids, 60(4) (2012), pp. 573-590.

[8] L. M. Blinov, Electro-Optical and Magneto-Optical Properties of Liquid Crystals, Wiley, 1983. 
[9] Juan-Pablo Borthagaray, Ricardo H. Nochetto, and Shawn W. Walker, $A$ structure-preserving FEM for the uniaxially constrained $\mathbf{Q}$-tensor model of nematic liquid crystals, Numerische Mathematik, 145 (2020), pp. 837-881.

[10] F. BRochard, L. LÉGer, AND R. B. MEYER, Freedericksz transition of a homeotropic nematic liquid crystal in rotating magnetic fields, J. Phys. Colloques, 36(C1) (1975), pp. 209-213.

[11] Miguel Camacho-Lopez, Heino Finkelmann, Peter PalfFy-Muhoray, and Michael SHelley, Fast liquid-crystal elastomer swims into the dark, Nature Materials, 3(5) (2004), pp. 307-310.

[12] Sylver CARTer, Amit Rotem, AND ShaWn W. WAlker, A domain decomposition approach to accelerate simulations of structure preserving nematic liquid crystal models, J. Non-Newtonian Fluid Mech., 283 (2020), 104335.

[13] P. G. CiARlet AND P.-A. RAVIART, Maximum principle and uniform convergence for the finite element method, Comput. Methods Appl. Mech. Eng., 2(1) (1973), pp. 17-31.

[14] Harry COles AND STEPHEN MORRIS, Liquid-crystal lasers, Nature Photonics, 4(10) (2010), pp. 676-685.

[15] P. DAsGupta, M. K. DAS, AND B. DAS, Fast switching negative dielectric anisotropic multicomponent mixtures for vertically aligned liquid crystal displays, Materials Research Express, 2(4) (2015), 045015.

[16] T. DAVIS AND E. GARTLAND, Finite element analysis of the Landau-de Gennes minimization problem for liquid crystals, SIAM J. Numer. Anal., 35(1) (1998), pp. 336-362.

[17] P. G. De Gennes And J. Prost, The Physics of Liquid Crystals, volume 83 of International Series of Monographs on Physics, Oxford Science Publication, Oxford, UK, 2nd edition, 1995.

[18] WIM H. DE JEU, Liquid Crystal Elastomers: Materials and Applications, Advances in Polymer Science, Springer, 2012.

[19] M. J. FreISER, Ordered states of a nematic liquid, Phys. Rev. Lett., 24(19) (1970), 1041.

[20] J. W. GoodBY, Handbook of Visual Display Technology (Editors: Chen, Janglin, Cranton, Wayne, Fihn, Mark), chapter Introduction to Defect Textures in Liquid Crystals, pages 12901314, Springer, 2012.

[21] Yuedong GU AND Nicholas L. AвBOtT, Observation of saturn-ring defects around solid microspheres in nematic liquid crystals, Phys. Rev. Lett., 85 (2000), pp. 4719-4722.

[22] JOON HeO, JAE-WON HUH, AND TAE-HOON YOON, Fast-switching initially-transparent liquid crystal light shutter with crossed patterned electrodes, AIP Adv., 5(4) (2015), 047118.

[23] Johan Hoogboom, Johannes A. A. W. Elemans, Alan E. Rowan, Theo H. M. RasING, AND ROELAND J. M. NOLTE, The development of self-assembled liquid crystal display alignment layers, Philosophical Transactions of the Royal Society of London A: Mathematical, Physical and Engineering Sciences, 365(1855) (2007), pp. 1553-1576.

[24] M. HumAR AND I. MUŠEVIČ, $3 d$ microlasers from self-assembled cholesteric liquid-crystal microdroplets, Opt. Express, 18(26) (2010), pp. 26995-27003.

[25] KAI JiAng, WeI Si, CHANG CHEN, AND CHENGLONG BAO, Efficient numerical methods for computing the stationary states of phase field crystal models, SIAM J. Sci. Comput., 42(6) (2020), pp. B1350-B1377.

[26] Sergey Korotov, Michal KřížEK, And PeKKa NeitTAanmÄKia, Weakened acute type condition for tetrahedral triangulations and the discrete maximum principle, Math. Comput., 70(233) (2001), pp. 107-119.

[27] Michal KŘížEK AND JAKUb ŠOlC, Acute versus nonobtuse tetrahedralizations, in M. Křížek, P. Neittaanmäki, S. Korotov, and R. Glowinski, editors, Conjugate Gradient Algorithms and 
Finite Element Methods, Scientific Computation, pages 161-170, Springer Berlin Heidelberg, 2004.

[28] JAN P. F. LAGERWALl AND GIUSy SCALIA, A new era for liquid crystal research: Applications of liquid crystals in soft matter nano-, bio- and microtechnology, Current Appl. Phys., 12(6) (2012), pp. 1387-1412.

[29] XAVIER LAMY, Uniaxial symmetry in nematic liquid crystals, Annales de l'Institut Henri Poincaré C, Analyse non linéaire, 32(5) (2015), pp. 1125-1144.

[30] Gi-Dong LeE, JAmes Anderson, AND PhiliP J. Bos, Fast q-tensor method for modeling liquid crystal director configurations with defects, Appl. Phys. Lett., 81(21) (2002), pp. 39513953.

[31] Teresa Lopez-LeOn And Alberto FernandeZ-Nieves, Drops and shells of liquid crystal, Colloid and Polymer Science, 289(4) (2011), pp. 345-359.

[32] L. A. Madsen, T. J. Dingemans, M. NAKATA, AND E. T. SAMulSKI, Thermotropic biaxial nematic liquid crystals, Phys. Rev. Lett., 92 (2004), 145505.

[33] Apala MAJUMdaR, Equilibrium order parameters of nematic liquid crystals in the landau-de gennes theory, Euro. J. Appl. Math., 21(2) (2010), pp. 181-203.

[34] ApAla MAJUMDAR AND ARGHIR ZARNESCU, Landau-de gennes theory of nematic liquid crystals: the oseen-frank limit and beyond, Arch. Rational Mech. Anal., 196(1) (2010), pp. 227-280.

[35] Gianni Dal Maso, Marco Forti, Mario Miranda, Sergio A. Spagnolo, and Luigi Ambrosio, Selected Papers, Springer Collected Works in Mathematics, Ennio De Giorgi, 2006.

[36] N. J. Mottram And C. J. P. NeWton, Introduction to Q-tensor theory, ArXiv e-prints, (2014).

[37] IGOR MUŠEVIČ AND SLOBODAN ŽUMER, Liquid crystals: Maximizing memory, Nature Materials, 10(4) (2011), pp. 266-268.

[38] ARTEM NAPOV AND YVAN NOTAY, Algebraic analysis of aggregation-based multigrid, Numer. Linear Algebra Appl., 18(3) (2011), pp. 539-564.

[39] ARTEM NAPOV AND YVAN NOTAY, An algebraic multigrid method with guaranteed convergence rate, SIAM J. Sci. Comput., 34(2) (2012), pp. A1079-A1109.

[40] YURII E. NESTEROV, A method of solving a convex programming problem with convergence rate $o\left(1 / k^{2}\right)$, Dokl. Akad. Nauk SSSR, 269 (1983), pp. 543-547.

[41] LUC NGUYEN AND ARGHIR ZARNESCU, Refined approximation for minimizers of a landau-de gennes energy functional, Calculus of Variations and Partial Differential Equations, 47(1-2) (2013), pp. 383-432.

[42] Jorge Nocedal And Stephen J. Wright, Numerical Optimization, Springer Series in Operations Research, Springer, 2nd edition, 2006.

[43] Ricardo H. Nochetto, Shawn W. Walker, and Wujun Zhang, Numerics for liquid crystals with variable degree of orientation, in Symposium NN-Mathematical and Computational Aspects of Materials Science, volume 1753 of MRS Proceedings, (2015).

[44] Ricardo H. Nochetto, SHAWn W. WAlker, AND WuJun ZHANG, A finite element method for nematic liquid crystals with variable degree of orientation, SIAM J. Numer. Anal., 55(3) (2017), pp. 1357-1386.

[45] Ricardo H. Nochetto, SHawn W. WAlker, AND Wujun ZHANG, The Ericksen model of liquid crystals with colloidal and electric effects, J. Comput. Phys., 352 (2018), pp. 568-601.

[46] P. PAlfFy-Muhoray, E. C. GARTland, AND J. R. Kelly, A new configurational transition in inhomogeneous nematics, Liquid Crystals, 16(4) (1994), pp. 713-718.

[47] B. T. POLYAK, Some methods of speeding up the convergence of iteration methods, USSR Compu- 
tational Mathematics and Mathematical Physics, 4(5) (1964), pp. 1-17.

[48] Veena Prasad, Shin-WoOng KANG, KA Suresh, LeEla Joshi, QingBing WANG, AND SATYENDRA KUMAR, Thermotropic uniaxial and biaxial nematic and smectic phases in bent-core mesogens, J. American Chem. Soc., 127(49) (2005), pp. 17224-17227.

[49] MiHA RAVNIK AND SLOBODAN ŽUMER, Landau-degennes modelling of nematic liquid crystal colloids, Liquid Crystals, 36(10-11) (2009), pp. 1201-1214.

[50] ANDRAŽ REŠETIČ, JERnEJA MilaVEC, BLAŽ ZuPANČič, VALENTINA DOMENICI, AND BOŠTJAN ZALAR, Polymer-dispersed liquid crystal elastomers, Nature Commun., 7 (2016), 13140.

[51] Aayush A. Shah, Heekyoung Kang, Kevin L. Kohlstedt, Kyung Hyun Ahn, Sharon C. Glotzer, Charles W. Monroe, And Michael J. Solomon, Self-assembly: Liquid crystal order in colloidal suspensions of spheroidal particles by direct current electric field assembly (small 10/2012), Small, 8(10) (2012), pp. 1551-1562.

[52] J. SHEN AND X. YANG, Numerical approximations of Allen-Cahn and Cahn-Hilliard equations, Discrete Contin. Dyn. Syst., 28(4) (2010), pp. 1669-1691.

[53] J. SHEN AND X. YANG, A phase-field model and its numerical approximation for two-phase incompressible flows with different densities and viscosities, SIAM J. Sci. Comput., 32(3) (2010), pp. 1159-1179.

[54] A. SOnNet, A. KILIAN, AND S. Hess, Alignment tensor versus director: description of defects in nematic liquid crystals, Phys. Rev. E, 52 (1995), pp. 718-722.

[55] André M. SONNET AND EPIFAnio VirGA, Dissipative Ordered Fluids: Theories for Liquid Crystals, Springer, 2012.

[56] Gilbert Strang AND GeOrge Fix, An Analysis of the Finite Element Method, WellesleyCambridge, 2nd edition, 2008.

[57] Jian Sun, Huihui Wang, Ling Wang, Hui CaO, Hui Xie, Xueyao luo, Jiumei XiaO, HangJun Ding, ZHOU YANG, AND HuAI YANG, Preparation and thermo-optical characteristics of a smart polymer-stabilized liquid crystal thin film based on smectic A-chiral nematic phase transition, Smart Materials and Structures, 23(12) (2014), 125038.

[58] SiMON ČOPAR, UROŠ TKALEC, IGOR MUŠEviČ, AND SlOBODAN ŽUMER, Knot theory realizations in nematic colloids, Proceedings of the National Academy of Sciences, 112(6) (2015), pp. 1675-1680.

[59] E. G. VIRGA, Variational Theories for Liquid Crystals, volume 8, Chapman and Hall, London, 1st edition, 1994.

[60] SHAWN W. WALKER, FELICITY: A Matlab/C++ toolbox for developing finite element methods and simulation modeling, SIAM J. Sci. Comput., 40(2) (2018), pp. C234-C257.

[61] Mingsheng WAng, Le He, Serkan Zorba, And Yadong Yin, Magnetically actuated liquid crystals, Nano Lett., 14(7) (2014), pp. 3966-3971, PMID: 24914876.

[62] Taylor H. Ware, Michael E. McConney, Jeong Jae Wie, Vincent P. Tondiglia, AND Timothy J. White, Voxelated liquid crystal elastomers, Science, 347(6225) (2015), pp. 982-984.

[63] Jonathan K. Whitmer, Xiaoguang Wang, Frederic Mondiot, Daniel S. Miller, Nicholas L. ABbotT, AND JuAN J. DE PABLO, Nematic-field-driven positioning of particles in liquid crystal droplets, Phys. Rev. Lett., 111 (2013), 227801.

[64] S. M. WISE, C. WANG, AND J. S. LOWENGRUB, An energy-stable and convergent finitedifference scheme for the phase field crystal equation, SIAM J. Numer. Anal., 47(3) (2009), pp. 2269-2288.

[65] L. J. YU AND A. SAUPE, Observation of a biaxial nematic phase in potassium laurate-1-decanol- 
water mixtures, Phys. Rev. Lett., 45(12) (1980), 1000.

[66] JIA ZHAO AND QI WANG, Semi-discrete energy-stable schemes for a tensor-based hydrodynamic model of nematic liquid crystal flows, J. Sci. Comput., 68(3) (2016), pp. 1241-1266.

[67] JiA ZhaO, XiAOfENG YANG, YUEZHENG GONG, AND QI WANG, A novel linear second order unconditionally energy stable scheme for a hydrodynamic Q-tensor model of liquid crystals, Comput. Methods Appl. Mech. Eng., 318 (2017), pp. 803-825.

[68] Jia ZHAO, XIAOFeNG YANG, JIE SHEN, AND QI WANG, A decoupled energy stable scheme for a hydrodynamic phase-field model of mixtures of nematic liquid crystals and viscous fluids, J. Comput. Phys., 305 (2016), pp. 539-556.

[69] Wei Zhu, Michael Shelley, and Peter Palffy-Muhoray, Modeling and simulation of liquid-crystal elastomers, Phys. Rev. E, 83 (2011), 051703. 